\title{
A Positive Feedback Loop of IncRNA-RMRP/ZNRF3 axis and Wnt/ $\beta$-catenin Signaling Regulates Temozolomide Resistance in Glioma
}

Tie Liu

Beijing Tiantan Hospital

Jie Hu

Beijing Tiantan Hospital

Bo Han

Beijing Tiantan Hospital

Shishan Tan

Beijing Tiantan Hospital

Wenqing Jia

Beijing Tiantan Hospital

Yu Xin ( $\nabla$ xy18801367416@163.com )

Beijing Tiantan Hospital

\section{Research}

Keywords: Long non-coding RNA, RMRP, temozolomide, resistance, IGF2BP3, ZNRF3, Wnt, $\beta$-catenin, glioma

Posted Date: December 4th, 2020

DOl: https://doi.org/10.21203/rs.3.rs-117711/v1

License: (c) (1) This work is licensed under a Creative Commons Attribution 4.0 International License. Read Full License 


\section{Abstract}

Background: Drug resistance strikingly limits the therapeutic effect of temozolomide (TMZ) (a common drug for glioma). Long non-coding RNA (IncRNA) RMRP was found to be implicated in glioma progression. However, the effects of RMRP on TMZ resistance along with related molecular mechanisms are poor defined in glioma.

Methods: RMRP, ZNRF3, and IGF2BP3 were screened out by bioinformatics analysis. The expression levels of IncRNAs and mRNAs were measured by RT-qPCR assay. Protein levels of genes were detected by western blot and immunofluorescence assays. ZNRF3 mRNA stability was analyzed using Actinomycin D assay. Cell proliferative ability and survival rate were determined by CCK-8 assay. Cell apoptotic patterns were estimated by flow cytometry. The effects of RMRP knockdown on the growth of TMZ-treated glioma xenograft tumors were explored in vivo. The relationships among IGF2BP3, RMRP and ZNRF3 were explored by bioinformatics prediction analysis, RNA immunoprecipitation and RNA pull-down assays.

Results: RMRP was highly expressed in glioma. RMRP knockdown curbed cell proliferation, facilitated cell apoptosis and reduced TMZ resistance in glioma cells and hindered the growth of TMZ-treated glioma xenograft tumors. RMRP exerted its functions by down-regulating ZNRF3 in glioma cells. IGF2BP3 interacted with RMRP and ZNRF3 mRNA. RMRP reduced ZNRF3 expression and mRNA stability by IGF2BP3. RMRP knockdown inhibited $\beta$-catenin expression by up-regulating ZNRF3 and $\beta$-catenin promoted RMRP expression in glioma cells.

Conclusion: RMRP/ZNRF3 axis and Wnt/ $\beta$-catenin signaling formed a positive feedback loop to regulate TMZ resistance in glioma. The sustained activation of Wnt/ $\beta$-catenin signaling by RMRP contributes the better management of cancers.

\section{Background}

Glioma is the commonest type of malignant brain and central nervous system tumors, accounting for approximately $80 \%$ of all malignant intracranial tumor cases [1]. More than $50 \%$ of glioma cases are diagnosed at the late stage (WHO grade IV glioblastoma), while glioblastoma patients after routine treatments have a poor prognosis with the 5-year survival rate of less than $5 \%$ [1, 2]. In addition, the recurrence rate of glioma is extremely high, and most of low-grade glioma will recur post initial therapy and transform into high-grade glioma [3-5].

Chemotherapy is a common clinical therapeutic strategy for glioma [6, 7]. Temozolomide (TMZ), an orally bioavailable DNA alkylating agent, has been a backbone of glioma treatment for more than 20 years due to its ability to penetrate the blood-brain barrier $[8,9]$. TMZ exerts its anti-tumor activity mainly through inducing base mismatches, DNA repair aberration, DNA strand breaks and cell death [9]. However, TMZ only can slightly increase the survival of patients with glioblastoma and many patients with glioblastoma have poor or no responses to TMZ, which is mainly caused by inherent and acquired TMZ resistance [10, 
11]. Thus, it is imperative to clarify the molecular mechanisms of TMZ resistance and identify novel therapeutic targets to improve its therapeutic efficacy.

Long non-coding RNAs (IncRNAs) are a group of RNA transcripts longer than 200 nucleotides in length that lack protein-coding potential $[12,13]$. LncRNAs have been found to be involved in the tumor onset and progression of multiple malignancies including glioma $[13,14]$. Moreover, dysregulated IncRNAs in clinical glioma samples are closely associated with tumor grade, tumor malignant phenotypes and patients' survival, which have potential values in the diagnosis, treatment and prognosis of glioma [1416]. LncRNAs can exert their functions through regulating almost all aspects of gene expression such as RNA processing, mRNA stability/modification/translation $[15,17]$. N6-methyladenosine (m6A), the most abundant modification in eukaryotic RNAs, has attracted much attention from researchers over the past decades due to its association with cancer development, progression and chemoresistance and its crucial roles in regulating almost all steps of RNA metabolism $[18,19]$. Additionally, emerging studies show that IncRNAs are involved in the regulation of m6A methylation on mRNAs by targeting m6A regulators in cancers [20,21]. Furthermore, accumulating RNA binding proteins (RBPs) with the binding activities with mRNAs and non-coding RNAs have been found to be crucial regulators in m6A-directed RNA metabolism [22]. For instance, IncRNA LINC00942 facilitated breast cancer cell proliferation and suppressed cell apoptosis by increasing mRNA stability and protein levels of C-X-C motif chemokine receptor 4 (CXCR4) and cytochrome P450 family 1 subfamily B member 1 (CYP1B1) via facilitating methyltransferase like 14 (METTL14)-mediated m6A methylation [23]. LncRNA Rho GTPase activating protein 5 (ARHGAP5) antisense RNA 1 (ARHGAP5-AS1) enhanced the resistance of gastric cancer cells to chemotherapeutic drugs (cisplatin, doxorubicin, and 5-fluorouracil) and elevated the m6A modification and stability of ARHGAP5 mRNA by recruiting methyltransferase like 3 (METTL3) to ARHGAP5 mRNA [24]. LncRNA RNA component of mitochondrial RNA processing endoribonuclease (RMRP) has been found to be an oncogenic factor in multiple cancers such as multiple myeloma [25], neonatal neuroblastoma [26] and non-small-cell lung cancer [27]. Also, a recent study demonstrated that RMRP was highly expressed in glioma tissues and RMRP knockdown led to the marked reduction of cell proliferative, migratory and invasive abilities and notable increase of cell apoptotic rate in glioma [28]. However, it remains unknown whether RMRP was involved in the regulation of TMZ resistance in glioma. In addition, the molecular mechanisms of RMRP underlying TMZ resistance have not been characterized.

In this text, we further investigated the effects of RMRP knockdown on glioma cell proliferation, apoptosis and TMZ resistance in vitro and the growth of TMZ-treated glioma xenograft tumors in vivo. Also, downstream target genes of RMRP and RBPs involved in the regulation of m6A modification of genes were further examined.

\section{Materials And Methods \\ Clinical samples}


Glioma tissues were obtained by surgical excision from glioma patients without any treatment $(n=20,20-$ 50 years old $)$ and age-matched patients with relapsed glioma after therapy $(n=12,20-50$ years old during July 2018 to December 2018 at Beijing Tiantan Hospital of Capital Medical University. Our study was approved by the Medical Ethics Committee of Beijing Tiantan Hospital of Capital Medical University. Also, written informed consents were obtained from all patients prior to experiments.

\section{Reagents}

Small interference RNAs (siRNAs) targeting RMRP, ZNRF3 and IGF2BP3 and a scramble control siRNA (siNC) were synthesized by GenePharma Co., Ltd. (Shanghai, China). Sense siRNA sequences were provided in Table 1. The pcDNA3.1-RMRP and $\beta$-catenin overexpression plasmid was purchased from Sangon Biotech Co., Ltd (Shanghai, China). TMZ were purchased from MedChemExpress Co., Itd. (Monmouth Junction, NJ, USA). In vitro experiments, TMZ was dissolved in DMSO.

\section{Cell culture and transfection}

U251 cells were purchased from Cell bank of Chinese Academy of Sciences (Shanghai, China) and were cultured in Dulbecco's Modified Eagle's Medium (DMEM) (Thermo Fisher Scientific, Rockford, IL, USA) supplemented with $10 \%$ fetal bovine serum (FBS, Thermo Fisher Scientific). LN229 cells were obtained from American Type Culture Collection (ATCC, Manassas, VA, USA) and were grown in DMEM medium (Thermo Fisher Scientific) containing 5\% FBS (Thermo Fisher Scientific). TMZ-resistant U251 cell line (U251/TMZ) and TMZ-resistant LN229 cell line (LN229/TMZ) were established by continuous exposure to increasing doses of TMZ. All cells were maintained in a $95 \%$ air $/ 5 \% \mathrm{CO}_{2}$ incubator at $37^{\circ} \mathrm{C}$. SiRNAs or plasmids, alone or in combination, were transfected into U251 and LN229 cells using Lipofectamine 3000 reagent (Thermo Scientific) following the protocols of manufacturer.

\section{RT-qPCR assay}

Total RNA was extracted from glioma tissues and cells using Trizol reagent (Thermo Fisher Scientific) following the instructions of manufacturer. Next, first strand CDNA was synthesized using SuperScript ${ }^{\mathrm{TM}}$ III Reverse Transcriptase (Thermo Fisher Scientific), random primers and RNA template. Subsequently, quantitative PCR reactions were performed using SYBR ${ }^{T M}$ Green PCR Master Mix (Thermo Scientific), cDNA template and specific PCR primers for RMRP, HOXA-AS3, CASC9, ZNRF3, and GAPDH. GAPDH acted as the house-keeping gene to normalize the expression of other genes. The quantitative PCR primer sequences were presented in Table 2.

\section{Western blot analysis}


U251 and LN229 cells were lysed using RIPA lysis buffer (Solarbio Life Sciences, Beijing, China) containing protease inhibitor cocktail (Sigma-Aldrich, St Louis, MO, USA). After high-speed centrifugation (12000rpm, $15 \mathrm{~min}, 4^{\circ} \mathrm{C}$ ), cell supernatants were collected. The concentration of protein in cell supernatants was measured using Bio-Rad Bradford Protein Assay Kit (Bio-Rad Laboratories, Hercules, CA, USA). Next, protein ( $30 \mu \mathrm{g} / \mathrm{sample}$ ) was separated by sodium dodecyl sulfate-polyacrylamide gel electrophoresis and transferred on nitrocellulose membranes (Millipore, Bedford, MA, USA). The membranes were sequentially incubated with $5 \%$ non-fat milk for $1 \mathrm{~h}$ at room temperature, primary antibody for $12 \mathrm{~h}$ at $4^{\circ} \mathrm{C}$ and corresponding horseradish peroxidase-conjugated secondary antibody for 1 $h$ at room temperature. Next, the membranes were exposed to the Pierce ${ }^{T M} E C L$ Western Blotting Substrate (Thermo Scientific) to detect the protein signals.

\section{CCK-8 assay}

Cell proliferative ability and survival rate were measured using CCK-8 kit (Beyotime Biotechnology). For the detection of cell proliferative activity, transfected cell suspensions $(100 \mu \mathrm{l} /$ well) were seeded into 96well plates and then co-incubated with CCK-8 solution ( $10 \mu \mathrm{l} /$ well) at $0,24,48,72 \mathrm{~h}$ after plating. After $3 \mathrm{~h}$ of co-incubation, the optical density (OD) values were measured at $450 \mathrm{~nm}$ using the SpectraMax 190 microplate reader (Molecular Device, San Jose, CA, USA). For the measurement of cell survival rate, transfected cells $(100 \mu \mathrm{l} /$ well) were seeded into 96 -well plates for $24 \mathrm{~h}$ and then treated with different concentrations $(8,16,32,64,128$, or $256 \mu \mathrm{M})$ of TMZ for another $24 \mathrm{~h}$. Next, $10 \mu \mathrm{l}$ of CCK-8 solution was added into each well. Three hours later, OD values were determined at $450 \mathrm{~nm}$, followed by the determination of $50 \%$ inhibitory concentration $\left(\mathrm{IC}_{50}\right)$. Cell survival rate $(\%)=(\mathrm{OD}$ (drug treated group)-OD (blank group)/OD (untreated group)-OD (blank group))×100\%.

\section{Cell apoptotic rate detection}

Cell apoptotic rate was estimated using the Annexin V-FITC Apoptosis Detection Kit (Beyotime Biotechnology) according to manufacturer's instructions. Briefly, cells were collected at $48 \mathrm{~h}$ after transfection and re-suspended in Annexin V-FITC binding solution. Next, cells were co-incubated with Annexin V-FITC and Propidium lodide (PI) solutions for $15 \mathrm{~min}$ at room temperature in a dark place. Next, stained samples were placed in an ice bath and cell apoptotic proportion was determined using a flow cytometry (BD Biosciences, San Diego, CA, USA).

\section{RNA immunoprecipitation (RIP) assay}

RIP assay was carried out in U251 cells using the Magna RIP RNA-Binding Protein Immunoprecipitation Kit (Millipore, Temecula, CA, USA) and antibody against IGF2BP3 or IgG following the manufacturer's protocols. RMRP and ZNRF3 mRNA levels enriched by IGF2BP3 or IgG antibody were measured through RT-qPCR assay. 


\section{RNA pull down assay}

RNA pull down assay was performed using a Pierce ${ }^{\text {TM }}$ Magnetic RNA-Protein Pull-Down Kit (Thermo Fisher Scientific) according to the manufacturer's protocols. Biotinylated ZNRF3 3' non-coding region (3'UTR) fragment 1 (1-2477 nt), fragment 2 (2445-3539 nt) and fragment 3 (3506-3912 nt) were purchased from Wuhan Genecreate CO., Itd. (Wuhan, China). Briefly, biotin-labeled RNAs were captured by streptavidinconjugated magnetic beads. Next, the RNA-bead complexes were co-incubated overnight at $4^{\circ} \mathrm{C}$ with the lysates of U251 cells. Finally, RNA-associated proteins were eluted and the protein level of IGF2BP3 was determined by western blot assay.

\section{mRNA stability analysis}

ZNRF3 mRNA stability was measured using actinomycin D (ActD, Sigma-Aldrich) assay. Briefly, $5 \mu \mathrm{g} / \mathrm{ml}$ of ActD as added into cell medium at $24 \mathrm{~h}$ post transfection. At $0,2,4,6 \mathrm{~h}$ after ActD treatment, RNA was isolated from cells and ZNRF3 mRNA level was tested through RT-qPCR assay.

\section{Subcellular localization analysis of RMRP}

Cytoplasmic and nuclear RNA was isolated from U251 and LN229 cells using Cytoplasmic \& Nuclear RNA Purification Kit (Norgen Biotek Inc, Thorold, Canada) following the protocols of manufacturer. Next, the levels of GAPDH, RMRP and U6 snRNA in cytoplasm and nucleus were determined through RT-qPCR assay.

\section{Immunofluorescence (IF) assay}

Cells were fixed with $4 \%$ paraformaldehyde solution for $15 \mathrm{~min}$ and then treated with $0.5 \%$ Triton X-100 solution for $20 \mathrm{~min}$. Next, samples on the slides were blocked with normal goat serum (BOSTER Biological Technology Co., Itd., Wuhan, China) for $30 \mathrm{~min}$ at room temperature and then incubated overnight with primary antibody against $\beta$-catenin at $4^{\circ} \mathrm{C}$. On the next day, samples were incubated with FITC-labeled goat anti-rabbit IgG (Ptoteintech Group, Inc., Wuhan, China) for $1 \mathrm{~h}$ at $37^{\circ} \mathrm{C}$ in the dark. Next, cell nuclei were stained with DAPI solution (Beyotime Biotechnology) for 5 min under the dark conditions. After mounted with Fluoromount-G (Birmingham, AL, USA), the slides were imaged using the Olympus BX53 microscope (Olympus Opticol Co. Itd., Tokyo, Japan).

\section{In vivo experiments}

Lentiviruses carrying interference fragment targeting RMRP (sh-RMRP) and lentiviruses bearing scramble control fragment (sh-NC) were obtained from Novobio Biotech Co., Itd. (Shanghai, China). U251 cells were 
infected with RMRP-targeting or non-targeting lentiviruses for 3 days and then screened with puromycin $(1 \mu \mathrm{g} / \mathrm{ml})$ for 1 week to establish cell lines with or without RMRP stable knockdown.

Mouse experiments were conducted with the approval of the Animal Care and Use Committee of Beijing Tiantan Hospital of Capital Medical University and performed with the standard experimental procedures. The BALB/C nude mice (male, 6 weeks old) were obtained from Laboratory Animal Center of of Beijing Tiantan Hospital of Capital Medical University and acclimatized to the new surroundings for 1 week. Mice were randomly divided into control, sh-NC and sh-RMRP groups ( $n=6 /$ group). U251/TMZ cells $\left(1 \times 10^{7}\right.$ cells/mouse), U251/TMZ cells infected with sh-NC ( $1 \times 10^{7}$ cells/mouse), U251/TMZ cells infected with sh-RMRP ( $1 \times 10^{7}$ cells/mouse) were injected subcutaneously into the right back of mice in the control, shNC and sh-RMRP groups. TMZ ( $25 \mathrm{mg} / \mathrm{kg}$ body weight) were intraperitoneally injected into mice every other day for 14 days when tumor volumes reached to approximately $100 \mathrm{~mm}^{3}$. TMZ was dissolved in $70 \%$ normal saline and 30\% DMSO. Tumor volume was monitored using a vernier caliper every 2 days and calculated using the following formula: tumor volume $\left(\mathrm{mm}^{3}\right)=\left(L \times W^{2}\right) / 2$, where $L$ and $W$ respectively represents tumor's length and width. Tumors were resected, photographed and weighed at the end of experiments.

\section{Statistical analysis}

Data were analyzed using GraphPad Prism 6 software (GraphPad Software Inc., La Jolla, CA, USA) with results presenting as means \pm standard deviation. Differential analysis between clinical samples was performed using paired t-tests. Differences between cell line data were compared using unpaired t-tests. Differences among groups were compared using one-way ANOVA or two-way ANOVA along with Bonferroni post hoc test. Differences were regarded as statistically significant when $P$ value was less than 0.05 .

\section{Results}

\section{Expression profile analysis of IncRNAs in glioma and screening of interested IncRNAs}

In this project, the expression data of IncRNAs in glioma $(n=168)$ and normal brain tissues $(n=150)$ were downloaded from TCGA and GTEx databases, respectively. Next, differentially expressed IncRNAs $\left(\left|\log _{2} \mathrm{FC}\right| \geq 1\right.$ and $P \otimes 0.05$ ) were identified in glioma samples versus normal brain tissue samples. The top 100 up-regulated IncRNAs in glioma tissues were presented in Fig. 1A. After literature retrieval, 3 IncRNAs (RMRP, HOXA-AS3, and CASC9) related to glioma tumorigenesis and progression were screened out for further investigations. Moreover, GEPIA database analyses revealed that expression levels of RMRP, HOXA-AS3, and CASC9 were notably up-regulated in glioma tissues than that in normal tissues (Fig. 1B). In view of the association of tumor recurrence and drug resistance, expression patterns of RMRP, HOXA- 
AS3, and CASC9 were examined in tumor tissues isolated from patients with primary glioma $(n=20)$ and recurrent glioma post therapy $(n=12)$. Results showed that RMRP and HOXA-AS3 expression levels were higher in recurrent group than that in primary group without any treatment (Fig. 1C-1E). To our knowledge, the association of HOXA-AS3 and drug resistance has been reported in a previous article [29]. However, no or few studies were performed to explore the correlation of RMRP and drug resistance in glioma. Hence, the roles and molecular basis of RMRP in glioma tumorigenesis and TMZ resistance were further investigated in the subsequent experiments.

\section{Effects of RMRP knockdown on glioma cell proliferation, apoptosis and TMZ resistance}

To have a deep insight into the functions of RMRP in glioma progression and TMZ resistance, 3 siRNAs targeting RMRP (si-RMRP\#1, si-RMRP\#2, and si-RMRP\#3) and a scramble control siRNA (si-NC) were synthesized. Subsequent transfection efficiency analyses revealed that the transfection of si-RMRP\#2 or si-RMRP\#3 could markedly reduce RMRP abundance in U251 and LN229 cells compared to si-NCtransfected group (Fig. 2A). Considering the best knockdown effect of si-RMRP\#3 on RMRP, si-RMRP\#3 was selected for following loss-of-function experiments. CCK-8 assay revealed that RMRP depletion strikingly weakened the proliferative ability of U251 and LN229 cells (Fig. 2B). Moreover, RMRP knockdown led to an approximately 2.75-fold increase in cell apoptotic percentage (Q1-UR+Q1-LR) in U251 cells (Fig. 2C). Also, an approximately 2.64 -fold increase in cell apoptotic rate was observed in siRMRP-transfected LN229 cells than that in si-NC-transfected cells (Fig. 2C). In addition, TMZ-resistant glioma cell lines (U251/TMZ and LN229/TMZ) were successfully constructed, as evidenced by the marked increase of TMZ IC 50 value in U251/TMZ and LN229/TMZ cells compared to corresponding parental cells (Fig. 2D). Our data also revealed that RMRP depletion led to the notable reduction of TMZ $I_{50}$ value in U251, LN229, U251/TMZ and LN229/TMZ cells (Fig. 2E and 2F), suggesting that RMRP loss weakened TMZ resistance in parental and TMZ-resistant glioma cells. In summary, these outcomes presented that RMRP knockdown curbed cell proliferation, facilitated cell apoptosis and reduced TMZ resistance in glioma cells.

\section{Effects of RMRP knockdown on the growth of TMZ-treated glioma xenograft tumors}

Next, in vivo experiments were performed to further investigate the effect of RMRP loss on glioma tumorigenesis in TMZ-treated glioma xenograft tumors. As presented in Fig. 3A-3C, tumor volume and tumor weight were remarkably reduced in glioma xenograft tumors derived from U251/TMZ cells infected with sh-RMRP lentiviruses compared to un-infection or sh-NC infection group. RT-qPCR assay further validated that RMRP level was markedly reduced in glioma xenograft tumors derived from U251/TMZ cells infected with sh-RMRP lentiviruses relative to sh-NC group (Fig. 3D). That was to say, RMRP knockdown hindered the growth of TMZ-treated glioma xenograft tumors in vivo. 


\section{ZNRF3 was a target of RMRP3}

Recently, emerging evidences show that IncRNAs can exert their biological activities through regulating gene expression [30]. To identify functionally related genes with RMRP, the differential expression patterns of genes in glioma $(n=168)$ and normal brain tissues $(n=150)$, whose expression data were respectively downloaded from TCGA and GTEx databases, were also analyzed. Also, co-expression relationships of RMRP and the top 50 down-regulated genes (Table 3 ) in glioma were predicted through GEPIA database. Results showed that expression levels of 13 genes were negatively associated with RMRP level $(P<0.05)$ in glioma (Table 4). Based on $P$ value $(P<0.01), 7$ genes whose expression was highly associated with RMRP level (TOMM6, ZNRF3, EEF1G, ADORA2A, KIAA0408, KREMEN1, XBP1) were pricked out for further explorations. Ualcan database analysis further revealed that Glioma patients with low ZNRF3 expression had a poor prognosis $(P=0.037)$, while expression of the other 6 genes was not associated with glioma patients' survival (Fig. 4A). These data suggested the potential regulatory relationships between RMRP and ZNRF3 in glioma. Subcellular localization analyses revealed that RMRP was located in the nuclear and cytoplasm of U251 and LN229 cells (Fig. 4B). In addition, RT-qPCR and western blot assays demonstrated that RMRP depletion led to the remarkable elevation of ZNRF3 mRNA and protein levels in U251 and LN229 cells (Fig. 4C). Furthermore, actinomycin D assay showed that RMRP loss enhanced the stability of ZNRF3 mRNA in U251 and LN229 cells (Fig. 4D). In a word, these outcomes showed that RMRP knockdown promoted ZNRF3 expression by improving ZNRF3 mRNA stability in glioma cells.

\section{ZNRF3 down-regulation weakened the effects of RMRP knockdown on glioma cell proliferation, apoptosis and TMZ resistance.}

Knockdown efficiency analyses revealed that the transfection of si-ZNRF3\#1 or si-ZNRF3\#3 led to the notable down-regulation of ZNRF3 mRNA expression in U251 and LN229 cells (Fig. 5A). In view of the stronger silence efficiency, si-ZNRF3\#3 was used in the following experiments. Next, our data showed that ZNRF3 knockdown markedly abrogated the detrimental effect of RMRP depletion on cell proliferation in U251 and LN229 cells (Fig. 5B). Moreover, ZNRF3 silence triggered a notable reduction in cell apoptotic percentage from $22.84 \%$ to $11.55 \%$ in U251 cells transfected with si-RMRP (Fig. 5C). Also, cell apoptotic percentage was reduced from $19.53 \%$ to $10.27 \%$ in si-RMRP-transfected LN229 cells following the introduction of si-ZNRF3 (Fig. 5C). Moreover, ZNRF3 loss abolished the inhibitory effect of RMRP knockdown on cell survival in TMZ-treated parental glioma cells (U251 and LN229) and TMZ-resistant glioma cells (U251/TMZ and LN229/TMZ) (Fig. 5D and 5E). Additionally, an obvious increase of TMZ $\mathrm{IC}_{50}$ value was observed in cells co-transfected with si-RMRP and si-ZNRF3 compared to cells transfected with si-RMRP alone (Fig. 5D and 5E). In summary, these data revealed that ZNRF3 knockdown weakened the effects of RMRP depletion on glioma cell proliferation, apoptosis and TMZ resistance. In other words, 
RMRP knockdown inhibited glioma cell proliferation, facilitated glioma cell apoptosis and weakened the resistance of glioma cells to TMZ through up-regulating ZNRF3.

\section{RMRP regulated ZNRF3 expression and mRNA stability through RBP IGF2BP3}

Prior studies showed that RBPs could regulate the functions of IncRNAs and coding genes through direct interactions with these RNA transcripts [31,32]. Thus, RBPs that had the probability to bind with RMRP and ZNRF3 mRNA were screened out through POSTAR database. Combined with the aforementioned differential gene expression data of TCGA/GTEx databases, 17 RBPs with the possibility to interact with both RMRP and ZNRF3 mRNA were found to be differentially expressed in glioma (Fig. 6A, Table 5). Among these RBPs, IGF2BP3 was picked out for further examinations in view of its highest expression in glioma tissues (Table 5). RIP assay coupled with RT-qPCR assay demonstrated that RMRP and ZNRF3 mRNA could be substantially enriched by IGF2BP3 antibody in U251 cells (Fig. 6B). Considering the central role of $3^{\prime} U T R$ region in determining mRNA stability, the essential sites in the $3^{\prime} U T R$ in controlling the binding activity between IGF2BP3 and ZNRF3 mRNA were further examined. POSTAR2 database revealed that there were 5 potential IGF2BP3 binding sites in the 3'UTR of ZNRF3 mRNA (Table 6). RNA pull down assay showed that IGF2BP3 protein could be largely pulled down by biotin-labeled ZNRF3 mRNA 3'UTR fragment 2 (2445-3539 nt), but could not be pulled down by biotin-labeled ZNRF3 mRNA 3'UTR fragment 1 (1-2477 nt) and fragment 3 (3506-3912 nt) (Fig. 6C). Because ZNRF3 mRNA 3'UTR fragment 1 contains IGF2BP3 binding site 1 and 2, fragment 2 contains binding site 2-4, fragment 3 covers the binding site 4 and 5, we believed that IGF2BP3 binding site 3 in ZNRF3 mRNA 3'UTR (chr22: 29056929-29056950, GCTTTTAAAATGAGGTCTAAAA) might mainly govern the binding activity of ZNRF3 mRNA 3'UTR and IGF2BP3 protein. Next, overexpression efficiency analyses showed that the transfection of pcDNA3.1-RMRP overexpression plasmid markedly improved RMRP expression in U251 and LN229 cells compared to pcDNA3.1 control group (Fig. 6D). Moreover, RT-qPCR and western blot assays demonstrated that RMRP overexpression led to the notable reduction in ZNRF3 mRNA and protein levels in U251 and LN229 cells (Fig. 6E). Additionally, ectopic expression of RMRP reduced ZNRF3 mRNA stability in U251 and LN229 cells (Fig. 6F). IGF2BP3 knockdown markedly alleviated the detrimental effects of RMRP on ZNRF3 expression and mRNA stability in U251 and LN229 cells (Fig. 6E and 6F). These outcomes showed that RMRP reduced ZNRF3 expression and mRNA stability through IGF2BP3 in glioma cells.

\section{RMRP knockdown reduced $\beta$-catenin expression by up- regulating ZNRF3 and $\beta$-catenin promoted RMRP expression in glioma cells.}


Next, western blot assay showed that ZNRF3 loss led to the notable increase of $\beta$-catenin protein level in U251 and LN229 cells (Fig. 7A). Also, enhanced $\beta$-catenin protein signals were observed in U251 and LN229 cells transfected with si-ZNRF3 compared with si-NC-transfected cells (Fig. 7B). In addition, western blot and IF assays showed that RMRP silence reduced $\beta$-catenin protein expression and attenuated $\beta$-catenin signal intensity, while ZNRF3 down-regulation markedly alleviated the inhibitory effects of RMRP loss on $\beta$-catenin expression in U251 and LN229 cells (Fig. 7C and 7D). These data suggested that RMRP activated Wnt/ $\beta$-catenin signaling pathway by down-regulating ZNRF3 in glioma cells. Additionally, a previous study showed that $\beta$-catenin increased RMRP expression level in HEK293 cells [33]. Thus, the effect of $\beta$-catenin overexpression or not on RMRP expression was further examined in our project. Our data revealed that $\beta$-catenin overexpression led to the notable up-regulation of RMRP level in U251 and LN229 cells (Fig. 7E). These outcomes suggested that RMRP overexpression could induce the constant activation of Wnt/ $\beta$-catenin signaling pathway in glioma cells.

\section{Discussion}

In this project, our data revealed that RMRP expression was markedly up-regulated in glioma tissues compared to normal tissues and RMRP knockdown inhibited glioma cell proliferation and promoted glioma cell apoptosis, which was in accordance with the previous study [28]. Moreover, our study was the first to substantiate that RMRP depletion reduced the resistance of glioma cells to TMZ in vitro and inhibited the growth of TMZ-treated glioma xenograft tumors in vivo.

Bioinformatics analysis revealed that ZNRF3 expression was notably down-regulated in glioma tissues versus normal tissues and was negatively associated with RMRP expression in glioma tissues. Moreover, patients with glioma exhibiting low ZNRF3 expression had a poor prognosis. ZNRF3 has been identified to be a potential tumor suppressor in several cancers such as papillary thyroid cancer [34], nasopharyngeal cancer [35] and lung cancer [36]. For instance, enforced expression of ZNRF3 inhibited cell proliferation, facilitated cell apoptosis through reducing $\beta$-catenin and TCF-4 expression in colorectal cancer [37]. To our knowledge, the roles of ZNRF3 in glioma progression and TMZ resistance along with related molecular mechanisms are not or poor characterized. Thus, we further investigated whether RMRP could regulate ZNRF3 expression in glioma. Our outcomes disclosed that RMRP knockdown led to the remarkable increase of ZNRF3 mRNA and protein expression and ZNRF3 mRNA stability in glioma cells. Additionally, ZNRF3 downregulation markedly weakened the effects of RMRP knockdown on glioma cell proliferation, apoptosis and TMZ resistance.

Considering the binding potential of RBPs and mRNAs/non-coding RNAs and the vital roles of m6A modification in regulating RNA metabolism including mRNAs, RBP IGF2BP3 involved in regulating m6A modification were screened out based on bioinformatics prediction analysis and previous data. IGF2BP3, also named as IMP3, is a member of insulin-like growth factor- 2 mRNA-binding protein family [38]. IGF2BP3 has been reported to be involved in the regulation of tumor cell fate (e.g. growth, survival, invasion, drug resistance) in multiple cancers [38, 39]. For instance, IGF2BP3 knockdown hindered glioma cell proliferation and cell cycle progression and inhibited glioma stem-like cell migration [40,41]. 
Moreover, glioma patients with higher IGF2BP3 expression had a shorter overall and disease-free survival, and IGF2BP3 expression was positively associated with glioma grades [42, 43]. In addition, some evidences showed that IGF2BP3 could exert its functions through regulating the stability, degradation, and translation of mRNAs [38, 39]. In this text, our data demonstrated that IGF2BP3 could bind with RMRP and ZNRF3 3'UTR, and RMRP inhibited ZNRF3 expression and reduced ZNRF3 mRNA stability by IGF2BP3.

ZNRF3, a membrane E3 ligase, also have been found to be a negative regulator of Wnt signaling pathway [44]. Wnt/ $\beta$-catenin pathway has been found to be aberrantly activated in plenty of cancers and to be involved in the regulation of cancer progression $[45,46]$. Also, previous studies showed that the activation of Wnt signaling pathway could enhance the resistance of cancer cells to chemotherapeutic drugs [47]. $W n t / \beta$-catenin signaling pathway has been found to be closely linked with the tumorigenesis and progression of glioma and to be involved in the regulation of TMZ resistance in glioma [48]. Hence, we further investigated whether RMRP/ZNRF3 axis could regulate Wnt/ $\beta$-catenin signaling pathway in glioma cells. Our results showed that ZNRF3 knockdown promoted $\beta$-catenin expression in glioma cells. Moreover, RMRP loss inhibited $\beta$-catenin expression by up-regulating ZNRF3 in glioma cells, suggesting that RMRP enhanced $\beta$-catenin expression by down-regulating ZNRF3 in glioma cells. Additionally, consistent with a previous report [33], we demonstrated that $\beta$-catenin could increase RMRP expression in glioma cells. These data suggested that up-regulation of RMRP could trigger the sustained activation of Wnt/ $\beta$-catenin signaling pathway, while the sustained activation of Wnt/ $\beta$-catenin pathway have been found to be closely linked with tumor initiation, progression, recurrence, immune escape and therapeutic resistance in cancers [49].

Taken together, our data demonstrated that RMRP was highly expressed in glioma tissues versus normal brain tissues. RMRP depletion hindered cell proliferation, induced cell apoptosis and reduced TMZ resistance in glioma cells and curbed the growth of glioma xenograft tumors in vivo. Molecular mechanism investigations revealed that RMRP exerted its functions by increasing ZNRF3 expression and ZNRF3 mRNA stability via IGF2BP3 in glioma. Also, our outcomes further revealed that RMRP/ZNRF3 axis regulated the development and TMZ resistance by controlling Wnt signaling pathway in glioma. To our knowledge, this is the first study to test the effect of RMRP on TMZ resistance in glioma and elucidate the molecular mechanisms of RMRP involved in the development and TMZ resistance of glioma. The aberrant expression of RMRP in untreated and recurrent glioma tissues and close link of RMRP and the progression and TMZ resistance of glioma suggests the potential values of RMRP in the diagnosis, prognosis and treatment of glioma and the management of TMZ resistance. Considering the vital roles of ZNRF3 in Wnt signaling and cancer progression, the further elaboration on ZNRF3 regulatory mechanisms can deepen our understanding on the pathogenesis of glioma.

\section{Conclusions}

Our data suggested that RMRP overexpression could induce the sustained activation of Wnt/ $\beta$-catenin signaling pathway, which has vital values in the management of tumor recurrence, immune escape and 


\section{Abbreviations}

LncRNA

long non-coding RNA; RMRP:RNA component of mitochondrial RNA processing endoribonuclease; ZNRF3:zinc and ring finger 3; TMZ:temozolomide; IGF2BP3:insulin like growth factor 2 mRNA binding protein 3; mRNA:messenger RNA; RT-qPCR:reverse transcription-quantitative PCR; WHO:World Health Organization; m6A:N6-methyladenosine; RBP:RNA binding protein; CXCR4:C-X-C motif chemokine receptor 4; CYP1B1:cytochrome P450 family 1 subfamily B member 1; METTL14:methyltransferase like 14; ARHGAP5:Rho GTPase activating protein 5; ARHGAP5-AS1:ARHGAP5 antisense RNA 1; METTL3:methyltransferase like 3; siRNA:Small interference RNA; si-NC:scramble control siRNA; DMEM:Dulbecco's Modified Eagle's Medium; FBS:fetal bovine serum; ATCC:American Type Culture Collection; U251/TMZ:TMZ-resistant U251 cell line; LN229/TMZ:TMZ-resistant LN229 cell line; HOXAAS3:HOXA cluster antisense RNA 3; CASC9:cancer susceptibility 9; GAPDH:glyceraldehyde-3-phosphate dehydrogenase; CCK-8:Cell Counting Kit-8; OD:optical density; PI:Propidium lodide; 3'UTR:3' non-coding region (3'UTR); ActD:actinomycin D; IF:immunofluorescence; FITC:fluorescein isothiocyanate; DAPI:4',6-

Diamidino-2-phenylindole, dihydrochloride; sh-RMRP:lentiviruses carrying interference fragment targeting RMRP; sh-NC:lentiviruses bearing scramble control fragment; L:length; W:width; ANOVA:analysis of variance; TCGA:The Cancer Genome Atlas; GTEx:The Genotype-Tissue Expression; $\log _{2}$ FC: $\log _{2}$ FoldChange; GEPIA:Gene Expression Profiling Interactive Analysis; IC ${ }_{50}$ :half maximal inhibitory concentration; TOMM6:translocase of outer mitochondrial membrane 6; EEF1G:eukaryotic translation elongation factor 1 gamma; ADORA2A:adenosine A2a receptor; KREMEN1:kringle containing transmembrane protein 1; XBP1:X-box binding protein 1 .

\section{Declarations}

\section{Ethics approval and consent to participate:}

All procedures performed in studies involving human participants were in accordance with the Medical Ethics Committee of Beijing Tiantan Hospital of Capital Medical University and with the 1964 Helsinki declaration and its later amendments or comparable ethical standards. Informed consent was obtained from all individual participants included in the study.

\section{Consent for publication:}

All authors consent for pulication.

\section{Competing interests:}


The authors declare that they have no financial conflicts of interest.

\section{Funding:}

Not applicable.

\section{Authors' contributions:}

TL designed and performed the experiments, wrote the manuscript. $\mathrm{JH}$ and $\mathrm{BH}$ contributed to experimental work and data analysis. ST and WJ conducted the experiments. YX revised the manuscript. All authors have read and approved the final manuscript.

\section{Acknowledgements:}

I would like to express my gratitude to all those who have helped me during the writing of this thesis. Also, I would like to thank Mr. TL, JH, BH, ST and WJ, who contributed to the research work.

\section{References}

1. Barnholtz-Sloan JS, Ostrom QT, Cote D. Epidemiology of Brain Tumors. Neurol Clin. 2018;36:395419.

2. Davis ME. Epidemiology and Overview of Gliomas. Semin Oncol Nurs. 2018;34:420-9.

3. Chen W, Lei C, Liu P, Liu Y, Guo X, Kong Z, Wang Y, Dai C, Wang Y, Ma W, Wang Y. Progress and Prospects of Recurrent Glioma: A Recent Scientometric Analysis of the Web of Science in 2019. World Neurosurg. 2020;134:e387-99.

4. Weller M, Cloughesy T, Perry JR, Wick W. Standards of care for treatment of recurrent glioblastomaare we there yet? Neuro Oncol. 2013;15:4-27.

5. Sanai N, Chang S, Berger MS. Low-grade gliomas in adults. J Neurosurg. 2011;115:948-65.

6. Donovan LE, Lassman AB. Chemotherapy Treatment and Trials in Low-Grade Gliomas. Neurosurg Clin N Am. 2019;30:103-9.

7. Chaul-Barbosa C, Marques DF. How We Treat Recurrent Glioblastoma Today and Current Evidence. Curr Oncol Rep. 2019;21:94.

8. Schreck KC, Grossman SA. Role of Temozolomide in the Treatment of Cancers Involving the Central Nervous System. Oncology (Williston Park). 2018;32:555-60, 569.

9. Strobel H, Baisch T, Fitzel R, Schilberg K, Siegelin MD, Karpel-Massler G, Debatin KM. Temozolomide and Other Alkylating Agents in Glioblastoma Therapy. 2019, 7.

10. Jiapaer S, Furuta T, Tanaka S, Kitabayashi T, Nakada M. Potential Strategies Overcoming the Temozolomide Resistance for Glioblastoma. Neurol Med Chir (Tokyo). 2018;58:405-21. 
11. Arora A, Somasundaram K. Glioblastoma vs temozolomide: can the red queen race be won? Cancer Biol Ther. 2019;20:1083-90.

12. de Oliveira JC, Oliveira LC, Mathias C, Pedroso GA, Lemos DS, Salviano-Silva A, Jucoski TS, LoboAlves SC, Zambalde EP, Cipolla GA, Gradia DF. Long non-coding RNAs in cancer: Another layer of complexity. 2019, 21:e3065.

13. Chi Y, Wang D, Wang J, Yu W, Yang J. Long Non-Coding RNA in the Pathogenesis of Cancers. Cells $2019,8$.

14. Xi J, Sun Q, Ma L, Kang J. Long non-coding RNAs in glioma progression. Cancer Lett. 2018;419:2039.

15. Peng Z, Liu C, Wu M. New insights into long noncoding RNAs and their roles in glioma. Mol Cancer. 2018;17:61.

16. Yan $Y$, Xu Z, Li Z, Sun L, Gong Z. An Insight into the Increasing Role of LncRNAs in the Pathogenesis of Gliomas. Front Mol Neurosci. 2017;10:53.

17. Yao RW, Wang Y, Chen LL. Cellular functions of long noncoding RNAs. 2019, 21:542-551.

18. Dai D, Wang H, Zhu L, Jin H, Wang X. N6-methyladenosine links RNA metabolism to cancer progression. Cell Death Dis. 2018;9:124.

19. Lence T, Paolantoni C, Worpenberg L, Roignant JY. Mechanistic insights into m(6)A RNA enzymes. Biochim Biophys Acta Gene Regul Mech. 2019;1862:222-9.

20. Dai F, Wu Y, Lu Y, An C, Zheng X, Dai L, Guo Y, Zhang L, Li H, Xu W, Gao W. Crosstalk between RNA m(6)A Modification and Non-coding RNA Contributes to Cancer Growth and Progression. Mol Ther Nucleic Acids. 2020;22:62-71.

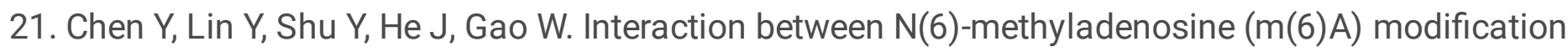
and noncoding RNAs in cancer. Mol Cancer. 2020;19:94.

22. Chen J, Fang X, Zhong P, Song Z, Hu X. N6-methyladenosine modifications: interactions with novel RNA-binding proteins and roles in signal transduction. RNA Biol. 2019;16:991-1000.

23. Sun T, Wu Z, Wang X, Wang Y, Hu X, Qin W, Lu S, Xu D, Wu Y, Chen Q, et al: LNC942 promoting METTL14-mediated $m(6) A$ methylation in breast cancer cell proliferation and progression. 2020 , 39:5358-5372.

24. Zhu L, Zhu Y, Han S, Chen M, Song P, Dai D, Xu W, Jiang T, Feng L. Impaired autophagic degradation of IncRNA ARHGAP5-AS1 promotes chemoresistance in gastric cancer. 2019, 10:383.

25. Xiao X, Gu Y, Wang G, Chen S. c-Myc, RMRP, and miR-34a-5p form a positive-feedback loop to regulate cell proliferation and apoptosis in multiple myeloma. Int J Biol Macromol. 2019;122:52637.

26. Pan J, Zhang D, Zhang J, Qin P, Wang J. LncRNA RMRP silence curbs neonatal neuroblastoma progression by regulating microRNA-206/tachykinin-1 receptor axis via inactivating extracellular signal-regulated kinases. Cancer Biol Ther. 2019;20:653-65. 
27. Wang Y, Luo X. Long noncoding RNA RMRP promotes proliferation and invasion via targeting miR-1$3 p$ in non-small-cell lung cancer. 2019, 120:15170-15181.

28. Feng W, Li L, Xu X, Jiao Y, Du W. Up-regulation of the long non-coding RNA RMRP contributes to glioma progression and promotes glioma cell proliferation and invasion. Arch Med Sci. 2017;13:1315-21.

29. Lin S, Zhang R, An X, Li Z, Fang C, Pan B, Chen W, Xu G, Han W. LncRNA HOXA-AS3 confers cisplatin resistance by interacting with HOXA3 in non-small-cell lung carcinoma cells. Oncogenesis. 2019;8:60.

30. Peng WX, Koirala P, Mo YY. LncRNA-mediated regulation of cell signaling in cancer. Oncogene. 2017;36:5661-7.

31. Xu J, Wang Z, Jin X, Li L, Pan T. Methods for Identification of Protein-RNA Interaction. Adv Exp Med Biol. 2018;1094:117-26.

32. Blagden S, Abdel Mouti M, Chettle J. Ancient and modern: hints of a core post-transcriptional network driving chemotherapy resistance in ovarian cancer. Wiley Interdiscip Rev RNA 2018, 9.

33. Park J, Jeong $S$. Wnt activated $\beta$-catenin and YAP proteins enhance the expression of non-coding RNA component of RNase MRP in colon cancer cells. Oncotarget. 2015;6:34658.

34. Qiu W, Yang Z, Fan Y, Zheng Q. ZNRF3 is downregulated in papillary thyroid carcinoma and suppresses the proliferation and invasion of papillary thyroid cancer cells. Tumour Biol. 2016;37:12665-72.

35. Wang Z, Wang $Y$, Ren $H$, Jin $Y$, Guo Y. ZNRF3 Inhibits the Invasion and Tumorigenesis in Nasopharyngeal Carcinoma Cells by Inactivating the Wnt/ $\beta$-Catenin Pathway. Oncol Res. 2017;25:571-7.

36. Shi J, Jiang X, Yu Z, He G, Ning H, Wu Z, Cai Y, Yu H, Chen A. ZNRF3 contributes to the growth of lung carcinoma via inhibiting Wnt/ $\beta$-catenin pathway and is regulated by miR-93. Tumour Biol. 2016;37:3051-7.

37. Yu N, Zhu H, Tao Y, Huang Y, Song X, Zhou Y, Li Y, Pei Q, Tan Q, Pei H. Association between prognostic survival of human colorectal carcinoma and ZNRF3 expression. Onco Targets Ther. 2016;9:6679-87.

38. Lederer M, Bley N, Schleifer C, Hüttelmaier S: The role of the oncofetal IGF2 mRNA-binding protein 3 (IGF2BP3) in cancer. In Seminars in cancer biology. Elsevier; 2014: 3-12.

39. Mancarella C, Scotlandi K. IGF2BP3 From Physiology to Cancer: Novel Discoveries, Unsolved Issues, and Future Perspectives. Frontiers in Cell and Developmental Biology 2019, 7.

40. Kouhkan F, Mobarra N, Soufi-Zomorrod M, Keramati F, Hosseini Rad SM, Fathi-Roudsari M, Tavakoli R, Hajarizadeh A, Ziaei S, Lahmi R, et al. MicroRNA-129-1 acts as tumour suppressor and induces cell cycle arrest of GBM cancer cells through targeting IGF2BP3 and MAPK1. J Med Genet. 2016;53:2433.

41. Bhargava S, Visvanathan A, Patil V, Kumar A, Kesari S, Das S, Hegde AS, Arivazhagan A, Santosh V, Somasundaram K. IGF2 mRNA binding protein 3 (IMP3) promotes glioma cell migration by 
enhancing the translation of RELA/p65. Oncotarget. 2017;8:40469-85.

42. Zhang GH, Zhong QY, Gou XX, Fan EX, Shuai Y, Wu MN, Yue GJ. Seven genes for the prognostic prediction in patients with glioma. Clin Transl Oncol. 2019;21:1327-35.

43. Del Gobbo A, Vaira V, Ferrari L, Patriarca C, Di Cristofori A, Ricca D, Caroli M: The oncofetal protein IMP3: a novel grading tool and predictor of poor clinical outcome in human gliomas. 2015, 2015:413897.

44. Hao HX, Jiang X, Cong F: Control of Wnt Receptor Turnover by R-spondin-ZNRF3/RNF43 Signaling Module and Its Dysregulation in Cancer. Cancers (Basel) 2016, 8.

45. Zhan T, Rindtorff N, Boutros M. Wnt signaling in cancer. 2017, 36:1461-1473.

46. Taciak B, Pruszynska I, Kiraga L, Bialasek M, Krol M. Wnt signaling pathway in development and cancer. J Physiol Pharmacol2018, 69.

47. Zhong Z, Virshup DM. Wnt Signaling and Drug Resistance in Cancer. 2020, 97:72-89.

48. He L, Zhou H, Zeng Z, Yao H, Jiang W, Qu H. Wnt/ $\beta$-catenin signaling cascade: A promising target for glioma therapy. J Cell Physiol. 2019;234:2217-28.

49. Martin-Orozco E, Sanchez-Fernandez A, Ortiz-Parra I, Ayala-San Nicolas M. WNT Signaling in Tumors: The Way to Evade Drugs and Immunity. Front Immunol. 2019;10:2854.

\section{Tables}

Table 1

Sense siRNA sequences of si-NC, si-RMRP\#1, si-RMRP\#2, siRMRP\#3, si-ZNRF3\#1, si-ZNRF3\#2, si-ZNRF3\#3, si-IGF2BP3\#1, siIGF2BP3\#2 and si-IGF2BP3\#3

\begin{tabular}{|ll|}
\hline si-NC & sense, 5'-UUCUCCGAACGUGUCACGUTT-3' \\
\hline si-RMRP\#1 & sense, 5'-CCUAGGCUACACACUGAGGACUTT-3' \\
\hline si-RMRP\#2 & sense, 5'-UGCUGAAGGCCUAUAUCCUTT-3' \\
\hline si-RMRP\#3 & sense, 5'-CCUAGGCUACACACUGAGGACUTT-3' \\
\hline si-ZNRF3\#1 & sense, 5'-GAAUAUUUAUUUACUAAUAUA-3' \\
\hline si-ZNRF3\#2 & sense, 5'-GAUAUUGUAUGUAAAUGUAAA-3' \\
\hline si-ZNRF3\#3 & sense, 5'-GCUGCUACACUGAGGACUATT-3' \\
\hline si-IGF2BP3\#1 & sense, 5'-AUAAAGUAUACAUUCUCACAG-3' \\
\hline si-IGF2BP3\#2 & sense, 5'-AUUAUACAGCGUCAAUUCCUG-3' \\
\hline si-IGF2BP3\#3 & sense, 5'-GGUACUAGCUAAGAAAUAAUU-3' \\
\hline
\end{tabular}


Table 2

Quantitative PCR primer sequences for GAPDH, RMRP, HOXA-AS3, CASC9, and ZNRF3.

\begin{tabular}{|ll|}
\hline GAPDH & F: 5'-GAAGGTGAAGGTCGGAGTC -3' \\
\hline IncRNA RMRP & F: 5'-GAAGATGGTGATGGGATTTC -3' \\
\hline IncRNA HOXA-AS3 & F:5' 5'-TGC GTAACTAGAGGGAGCTGAC-3' \\
\hline IncRNA CASC9 & F:5: 5'-ATTCAGCCAGAGAGTCAGCCACATTCATGGT-3' \\
\hline ZNRF3 & R:5'-AGTGCCAATGACTCTCCAGC-3' \\
\hline & F: 5'-TCCTTGTCAAAATCAAGCTGAAGC-3' \\
\hline R:5'-TGCTCTTGGAGTTGAACTTTCTG-3' \\
\hline
\end{tabular}


Table 3

Top 50 down-regulated genes in glioma tissues $(n=168)$ versus normal brain tissues $(n=150)$.

\begin{tabular}{|c|c|c|c|c|}
\hline gene_id & gene_name & gene_type & baseMean & log2FoldChange \\
\hline ENSG00000214736.7 & TOMM6 & protein_coding & 1374.204 & -13.9316 \\
\hline ENSG00000183762.12 & KREMEN1 & protein_coding & 1168.519 & -13.6978 \\
\hline ENSG00000213578.6 & CPLX3 & protein_coding & 881.104 & -13.1532 \\
\hline ENSG00000100219.16 & XBP1 & protein_coding & 650.0047 & -12.8518 \\
\hline ENSG00000243509.6 & TNFRSF6B & protein_coding & 563.3238 & -12.6451 \\
\hline ENSG00000213689.14 & TREX1 & protein_coding & 394.5534 & -12.1309 \\
\hline ENSG00000145863.11 & GABRA6 & protein_coding & 1263.159 & -11.0768 \\
\hline ENSG00000103310.10 & ZP2 & protein_coding & 259.5472 & -10.6913 \\
\hline ENSG00000254772.10 & EEF1G & protein_coding & 10691.19 & -10.0734 \\
\hline ENSG00000139574.8 & NPFF & protein_coding & 70.47618 & -9.64633 \\
\hline ENSG00000187664.9 & HAPLN4 & protein_coding & 788.5805 & -9.46491 \\
\hline ENSG00000204983.14 & PRSS1 & protein_coding & 66.5347 & -9.14992 \\
\hline ENSG00000259803.7 & SLC22A31 & protein_coding & 1122.359 & -9.14244 \\
\hline ENSG00000161992.6 & PRR35 & protein_coding & 905.7517 & -9.08997 \\
\hline ENSG00000140488.16 & CELF6 & protein_coding & 842.5066 & -8.94603 \\
\hline ENSG00000254852.8 & NPIPA2 & protein_coding & 41.43677 & -8.7939 \\
\hline ENSG00000275896.5 & PRSS2 & protein_coding & 59.96191 & -8.51508 \\
\hline ENSG00000169347.16 & GP2 & protein_coding & 44.80562 & -8.47527 \\
\hline ENSG00000140506.17 & LMAN1L & protein_coding & 222.1373 & -8.46198 \\
\hline ENSG00000183336.8 & BOLA2 & protein_coding & 30.36661 & -8.29844 \\
\hline ENSG00000124493.13 & GRM4 & protein_coding & 5095.998 & -8.26487 \\
\hline ENSG00000175535.6 & PNLIP & protein_coding & 41.79157 & -8.25373 \\
\hline ENSG00000086570.12 & FAT2 & protein_coding & 4351.549 & -8.17583 \\
\hline ENSG00000183379.9 & SYNDIG1L & protein_coding & 5193.95 & -8.1221 \\
\hline ENSG00000115665.9 & SLC5A7 & protein_coding & 70.7648 & -8.01853 \\
\hline ENSG00000128271.22 & ADORA2A & protein_coding & 1143.007 & -7.97506 \\
\hline ENSG00000189367.15 & KIAA0408 & protein_coding & 2062.133 & -7.9244 \\
\hline
\end{tabular}




\begin{tabular}{|c|c|c|c|c|}
\hline ENSG00000243789.11 & JMJD7 & protein_coding & 563.5629 & -7.89467 \\
\hline ENSG00000272573.6 & MUSTN1 & protein_coding & 302 & -7.82681 \\
\hline ENSG00000213927.4 & CCL27 & protein_coding & 22.78999 & -7.82074 \\
\hline ENSG00000101200.5 & AVP & protein_coding & 92.97653 & -7.80529 \\
\hline ENSG00000183579.16 & ZNRF3 & protein_coding & 973.3927 & -7.79792 \\
\hline ENSG00000188038.8 & NRN1L & protein_coding & 69.08776 & -7.77971 \\
\hline ENSG00000142789.20 & CELA3A & protein_coding & 24.23641 & -7.7586 \\
\hline ENSG00000100249.4 & C22orf31 & protein_coding & 15.80291 & -7.48736 \\
\hline ENSG00000170477.13 & KRT4 & protein_coding & 43.84427 & -7.34327 \\
\hline ENSG00000111245.15 & MYL2 & protein_coding & 59.22261 & -7.33423 \\
\hline ENSG00000137843.12 & PAK6 & protein_coding & 1470.04 & -7.32924 \\
\hline ENSG00000171195.11 & MUC7 & protein_coding & 21.18084 & -7.28611 \\
\hline ENSG00000094796.5 & KRT31 & protein_coding & 182.3399 & -7.22796 \\
\hline ENSG00000171772.16 & SYCE1 & protein_coding & 372.6 & -7.20241 \\
\hline ENSG00000243708.10 & PLA2G4B & protein_coding & 1990.801 & -7.19196 \\
\hline ENSG00000204001.9 & LCN8 & protein_coding & 206.1484 & -7.14751 \\
\hline ENSG00000118271.10 & TTR & protein_coding & 653.9319 & -7.08388 \\
\hline ENSG00000182450.13 & KCNK4 & protein_coding & 294.6109 & -7.00745 \\
\hline ENSG00000143032.8 & BARHL2 & protein_coding & 258.2312 & -6.97137 \\
\hline ENSG00000102924.12 & CBLN1 & protein_coding & 3189.46 & -6.83188 \\
\hline ENSG00000196689.12 & TRPV1 & protein_coding & 569.4355 & -6.81289 \\
\hline ENSG00000214338.10 & SOGA3 & protein_coding & 353.8444 & -6.79365 \\
\hline
\end{tabular}


Table 4

Correlation analyses of RMRP level and the top 50 down-regulated genes via GEPIA database.

\begin{tabular}{|c|c|c|c|c|}
\hline gene_id & gene_name & gene_type & $\mathrm{R}$ value & P-value \\
\hline ENSG00000214736.7 & TOMM6 & protein_coding & -0.29 & 0.00015 \\
\hline ENSG00000183579.16 & ZNRF3 & protein_coding & -0.29 & 0.00019 \\
\hline ENSG00000254772.10 & EEF1G & protein_coding & -0.22 & 0.0055 \\
\hline ENSG00000128271.22 & ADORA2A & protein_coding & -0.21 & 0.007 \\
\hline ENSG00000189367.15 & KIAA0408 & protein_coding & -0.21 & 0.0076 \\
\hline ENSG00000183762.12 & KREMEN1 & protein_coding & -0.2 & 0.0098 \\
\hline ENSG00000100219.16 & XBP1 & protein_coding & -0.2 & 0.0088 \\
\hline ENSG00000243789.11 & JMJD7 & protein_coding & -0.2 & 0.012 \\
\hline ENSG00000213689.14 & TREX1 & protein_coding & -0.19 & 0.017 \\
\hline ENSG00000254852.8 & NPIPA2 & protein_coding & -0.19 & 0.015 \\
\hline ENSG00000214338.10 & SOGA3 & protein_coding & -0.17 & 0.032 \\
\hline ENSG00000188038.8 & NRN1L & protein_coding & -0.16 & 0.048 \\
\hline ENSG00000137843.12 & PAK6 & protein_coding & -0.16 & 0.041 \\
\hline ENSG00000183379.9 & SYNDIG1L & protein_coding & -0.15 & 0.054 \\
\hline ENSG00000272573.6 & MUSTN1 & protein_coding & -0.15 & 0.055 \\
\hline ENSG00000140488.16 & CELF6 & protein_coding & -0.14 & 0.068 \\
\hline ENSG00000187664.9 & HAPLN4 & protein_coding & -0.12 & 0.13 \\
\hline ENSG00000243708.10 & PLA2G4B & protein_coding & -0.12 & 0.12 \\
\hline ENSG00000204001.9 & LCN8 & protein_coding & -0.11 & 0.16 \\
\hline ENSG00000182450.13 & KCNK4 & protein_coding & -0.11 & 0.17 \\
\hline ENSG00000171772.16 & SYCE1 & protein_coding & -0.1 & 0.18 \\
\hline ENSG00000196689.12 & TRPV1 & protein_coding & -0.097 & 0.22 \\
\hline ENSG00000243509.6 & TNFRSF6B & protein_coding & -0.082 & 0.3 \\
\hline ENSG00000145863.11 & GABRA6 & protein_coding & -0.07 & 0.38 \\
\hline ENSG00000175535.6 & PNLIP & protein_coding & -0.067 & 0.39 \\
\hline ENSG00000213927.4 & CCL27 & protein_coding & -0.065 & 0.41 \\
\hline
\end{tabular}




\begin{tabular}{|llllll|}
\hline ENSG00000213578.6 & CPLX3 & protein_coding & -0.064 & 0.42 \\
\hline ENSG00000171195.11 & MUC7 & protein_coding & -0.057 & 0.47 \\
\hline ENSG00000118271.10 & TTR & protein_coding & -0.056 & 0.48 \\
\hline ENSG0000000183336.8 & BOLA2 & protein_coding & -0.052 & 0.51 \\
\hline ENSG00000115665.9 & SLC5A7 & protein_coding & -0.047 & 0.55 \\
\hline ENSG00000169347.16 & GP2 & protein_coding & -0.045 & 0.59 \\
\hline ENSG00000139574.8 & NPFF & protein_coding & -0.044 & 0.58 \\
\hline ENSG00000101200.5 & AVP & protein_coding & -0.043 & 0.58 \\
\hline ENSG00000124493.13 & GRM4 & protein_coding & -0.041 & 0.61 \\
\hline ENSG00000204983.14 & PRSS1 & protein_coding & -0.04 & 0.64 \\
\hline ENSG00000170477.13 & KRT4 & protein_coding & -0.04 & 0.62 \\
\hline ENSG00000111245.15 & MYL2 & protein_coding & -0.04 & 0.54 \\
\hline ENSG00000094796.5 & KRT31 & protein_coding & -0.039 & 0.62 \\
\hline ENSG00000161992.6 & PRR35 & protein_coding & -0.038 & 0.63 \\
\hline ENSG00000259803.7 & SLC22A31 & protein_coding & -0.036 & 0.65 \\
\hline ENSG00000140506.17 & LMAN1L & protein_coding & -0.031 & 0.69 \\
\hline ENSG00000143032.8 & BARHL2 & protein_coding & -0.021 & 0.79 \\
\hline ENSG00000086570.12 & FAT2 & protein_coding & -0.01 & 0.9 \\
\hline ENSG00000103310.10 & ZP2 & protein_coding & 0.0035 & 0.96 \\
\hline ENSG00000102924.12 & CBLN1 & protein_coding & 0.018 & 0.82 \\
\hline ENSG00000275896.5 & PRSS2 & protein_coding & 0.025 & 0.75 \\
\hline ENSG00000142789.20 & CELA3A & protein_coding & 0.16 & 0.044 \\
\hline
\end{tabular}


Table 5

Expression patterns of 17 differentially expressed RBPs that had the likelihood to bind with both RMRP and ZNRF3 mRNA.

\begin{tabular}{|llll|}
\hline gene_name & gene_type & baseMean & log2FoldChange \\
\hline IGF2BP3 & protein_coding & 309.1855 & 7.126539 \\
\hline IGF2BP1 & protein_coding & 13.30504 & 4.005563 \\
\hline U2AF1 & protein_coding & 39.64123 & 2.069723 \\
\hline IGF2BP2 & protein_coding & 572.0013 & 1.969356 \\
\hline PTBP1 & protein_coding & 6228.647 & 1.66138 \\
\hline EIF4A3 & protein_coding & 2968.521 & 1.458592 \\
\hline XRN2 & protein_coding & 3305.495 & 1.37398 \\
\hline MSI1 & protein_coding & 1341.736 & 1.359946 \\
\hline SRSF9 & protein_coding & 2870.31 & 1.304158 \\
\hline SF3B4 & protein_coding & 2578.165 & 1.203024 \\
\hline SND1 & protein_coding & 6167.769 & 1.1116 \\
\hline NONO & protein_coding & 13010.02 & 1.086084 \\
\hline TNRC6A & protein_coding & 4124.074 & -1.06001 \\
\hline RANGAP1 & protein_coding & 6934.024 & -1.21977 \\
\hline RBFOX2 & protein_coding & 6642.03 & -1.58743 \\
\hline MBNL2 & protein_coding & 4176.68 & -1.6651 \\
\hline ELAVL3 & protein_coding & 7141.248 & -2.37595 \\
\hline
\end{tabular}

Table 6

Putative binding sites between IGF2BP3 and ZNRF3 3'UTR by POSTAR2 database.

\begin{tabular}{|lllll|}
\hline RBP & RBP info & Tissue type & Position & Genomic context \\
\hline IGF2BP3 & info & HEK293 & chr22:29055236-29055286 & UTR3 \\
\hline IGF2BP3 & info & HEK293 & chr22:29056067-29026099 & UTR3 \\
\hline IGF2BP3 & info & HEK293 & chr22:29056929-29056950 & UTR3 \\
\hline IGF2BP3 & info & HEK293 & chr22:29057129-29057161 & UTR3 \\
\hline IGF2BP3 & info & HEK293 & chr22:29057294-29057273 & UTR3 \\
\hline
\end{tabular}




\section{Figures}
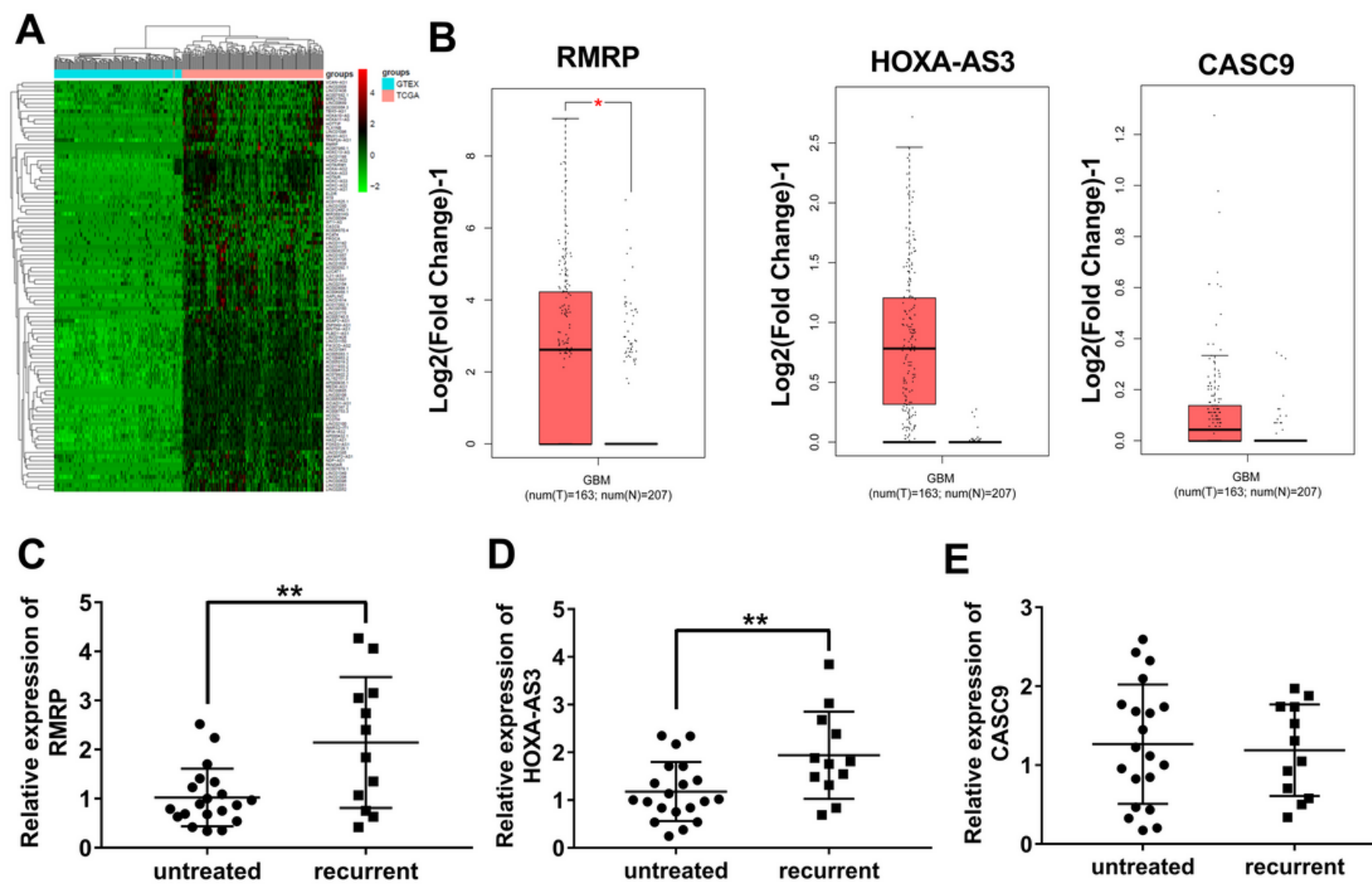

D

E
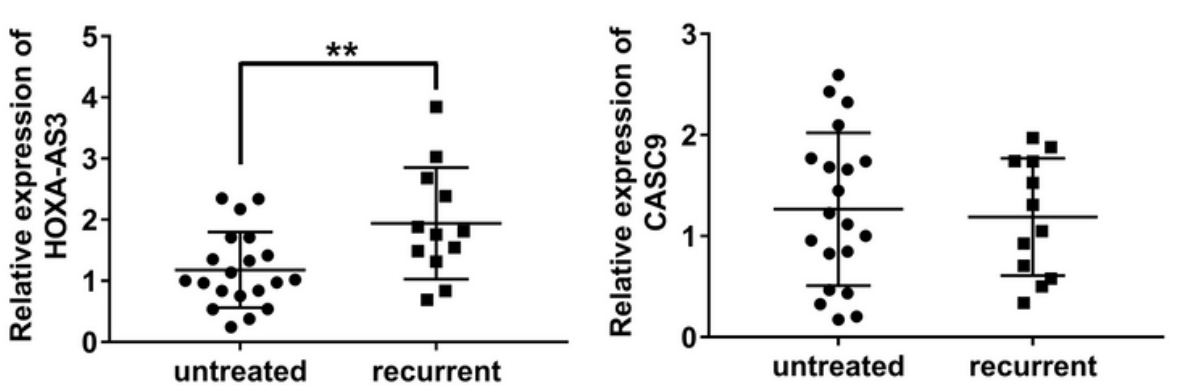

Figure 1

Expression profile analysis of IncRNAs in glioma and screening of interested IncRNAs. (A) The top 100 up-regulated IncRNAs in glioma group versus normal brain tissue group. (B) Expression analyses of 3 IncRNAs (RMRP, HOXA-AS3, and CASC9) in glioma versus normal tissues in GEPIA database. (C) Expression levels of RMRP, HOXA-AS3, CASC9 in tumor tissues isolated from patients with primary $(n=20)$ and recurrent $(n=12)$ glioma were measured through RT-qPCR assay. ${ }^{*} P<0.05 .{ }^{*} P<0.01$. 
A

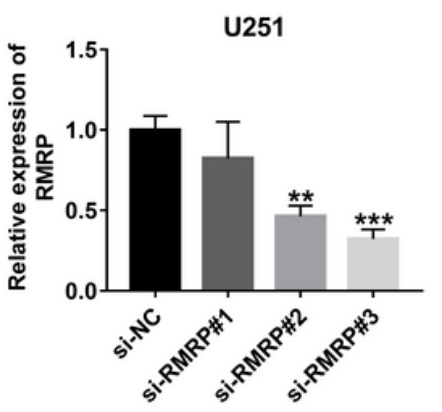

C

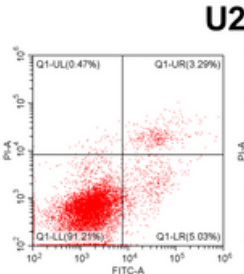

si-NC
si-RMRP

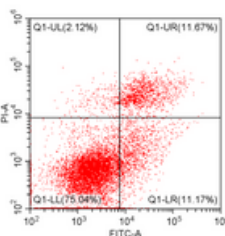

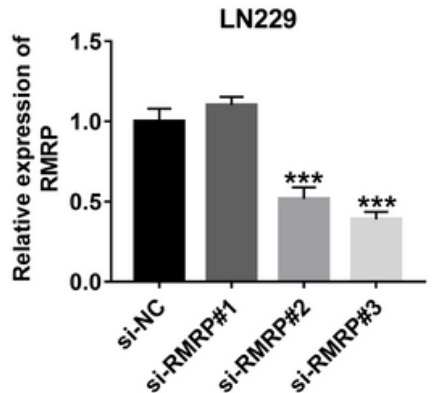

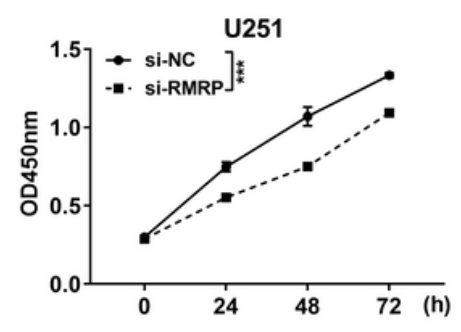

D

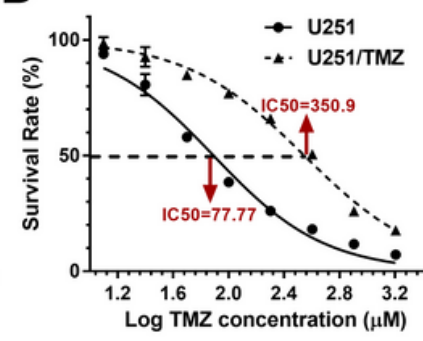

si-RMRP

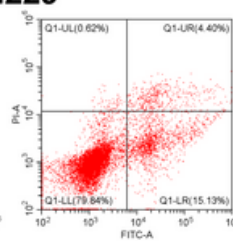

si-NC

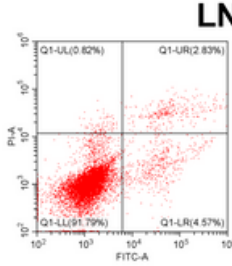

E

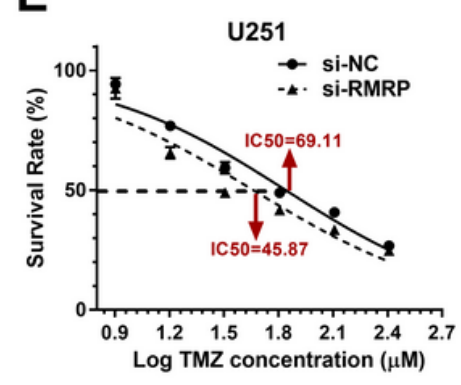

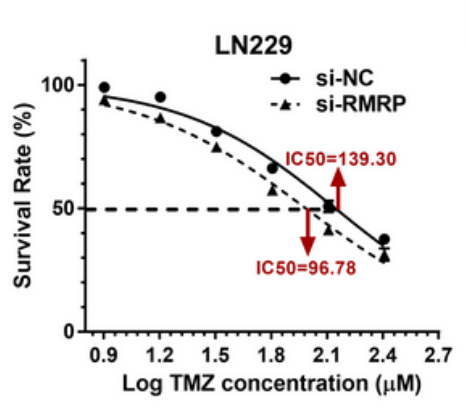

$\mathbf{F}$

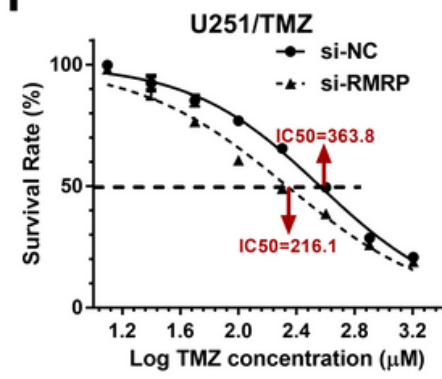

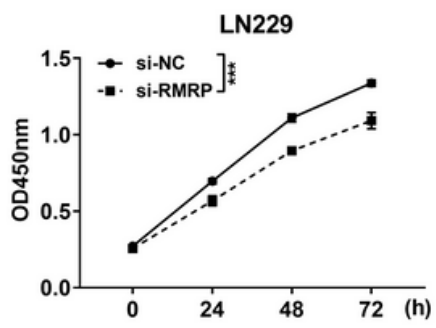

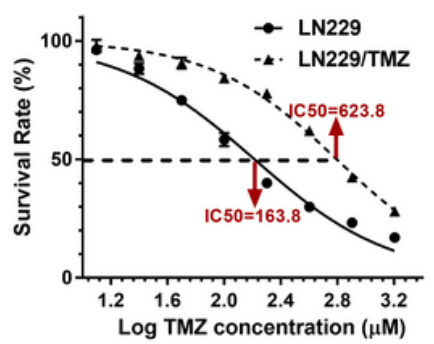

Figure 2

RMRP knockdown curbed cell proliferation, facilitated cell apoptosis and reduced TMZ resistance in glioma cells. (A) U251 and LN229 cells were transfected with si-NC, si-RMRP\#1, si-RMRP\#2, or siRMRP\#3. Forty-eight hours later, RMRP level was examined through RT-qPCR assay. (B) Cell proliferative ability was estimated through CCK-8 assay in U251 and LN229 cells transfected with si-NC or si-RMRP\#3 at $0,24,48,72 \mathrm{~h}$ post plating. (C) Cell apoptotic rate was determined by flow cytometry in U251 and LN229 cells transfected with si-NC or si-RMRP\#3 at $48 \mathrm{~h}$ after transfection. (D) U251, LN229, U251/TMZ and LN229/TMZ cells were treated with different doses of TMZ for $24 \mathrm{~h}$, followed by the measurement of TMZ IC50 values via CCK-8 assay. (E and F) U251, LN229, U251/TMZ and LN229/TMZ cells were transfected with si-NC or si-RMRP\#3. Transfected cells were seeded into 96-well plates for $24 \mathrm{~h}$ and then treated with different concentrations of TMZ for additional $24 \mathrm{~h}$. Next, cell survival rate was determined through CCK-8 assay. ${ }^{*} \mathrm{P}<0.05 .{ }^{* *} \mathrm{P}<0.01 .{ }^{*} * \mathrm{P}<0.001$. 
A

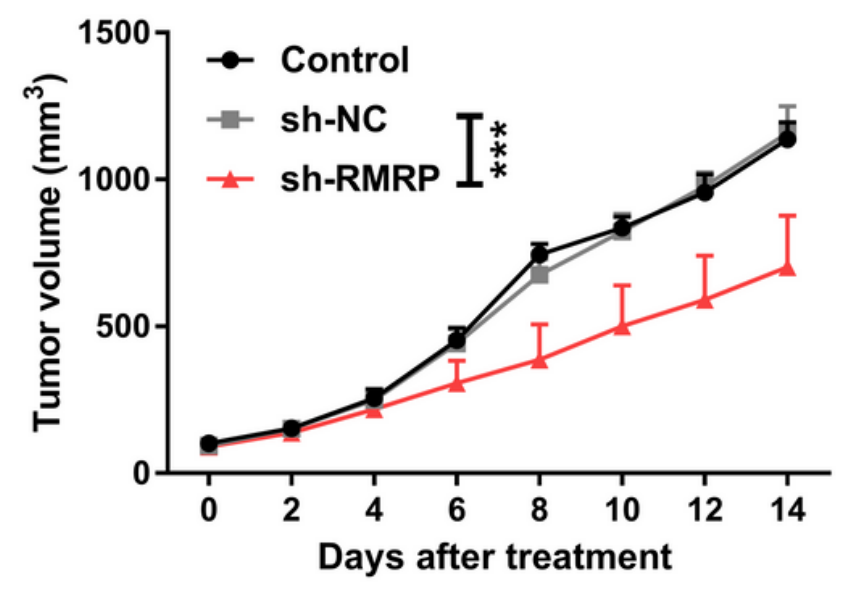

C

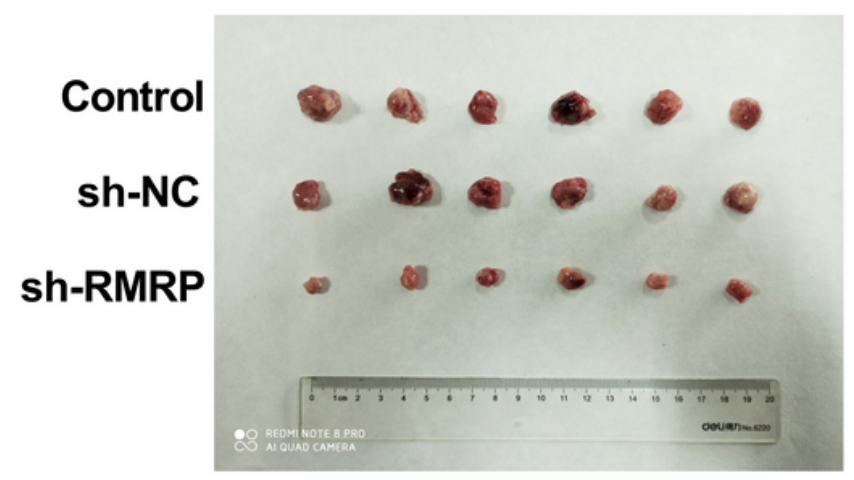

B

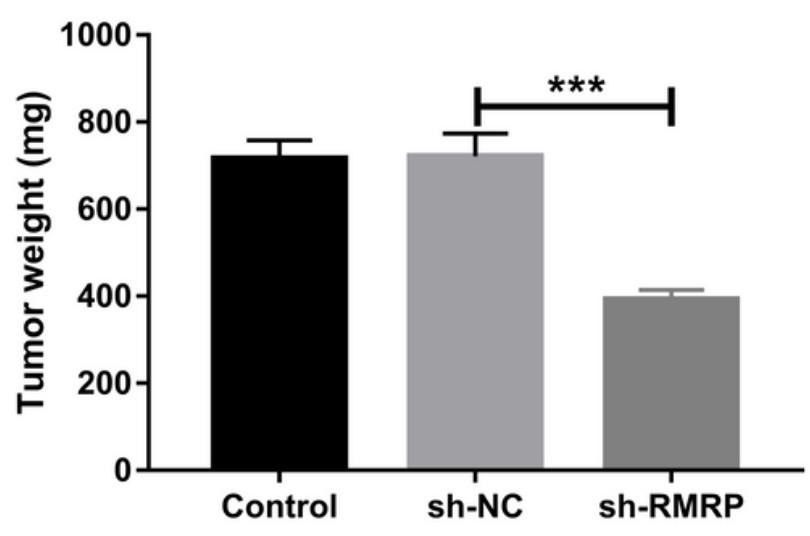

D

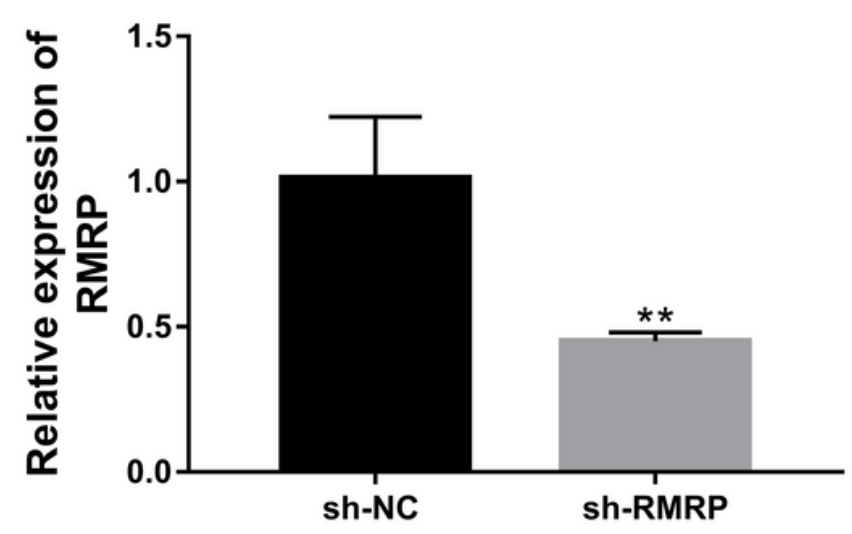

Figure 3

Effects of RMRP knockdown on the growth of TMZ-treated glioma xenograft tumors. (A) The volumes of xenograft tumors were measured every 2 days after TMZ treatment. (B) Tumor weight was determined on day 14 post TMZ treatment. (C) Representative photographs of xenograft tumors in control, sh-NC and sh-RMRP groups. (D) RMRP level was measured by RT-qPCR assay in xenograft tumors of sh-NC and shRMRP groups. ${ }^{* *} P<0.01 .{ }^{* * * P}<0.001$. 

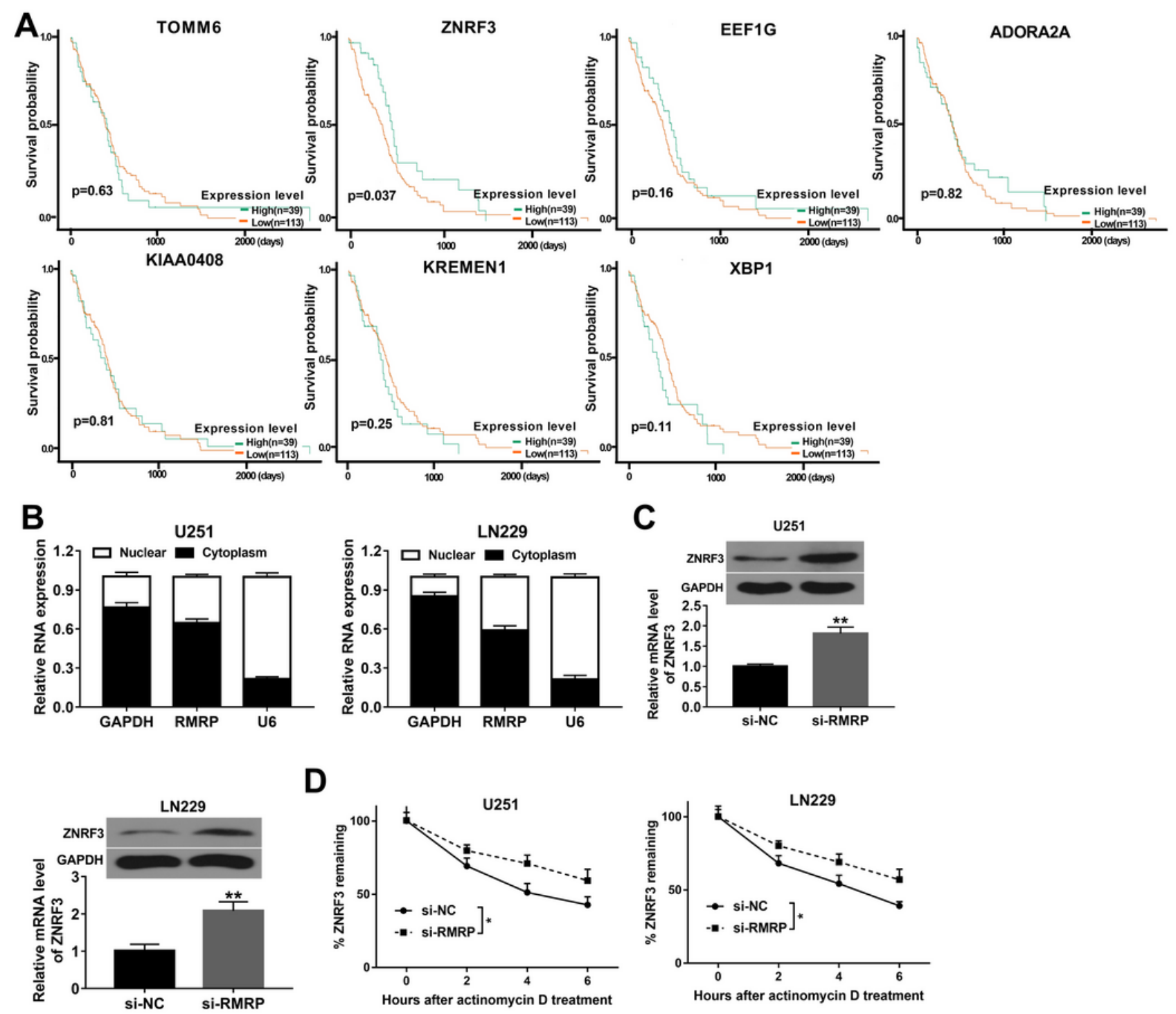

\section{Figure 4}

ZNRF3 was a target of RMRP3. (A) The survival analysis of glioma patients with high or low expression of TOMM6, ZNRF3, EEF1G, ADORA2A, KIAA0408, KREMEN1, XBP1. (B) RMRP level in the nucleus and cytoplasm of U251 and LN229 cells was measured by RT-qPCR assay after the isolation of nuclear and cytoplasmic RNA. (C) The effects of RMRP knockdown on ZNRF3 mRNA and protein expression were measured by RT-qPCR and western blot assays at $48 \mathrm{~h}$ after transfection in U251 and LN229 cells. (D) The effect of RMRP knockdown on ZNRF3 mRNA stability was examined through actinomycin D and RTqPCR assays in U251 and LN229 cells. **P<0.01. 
A
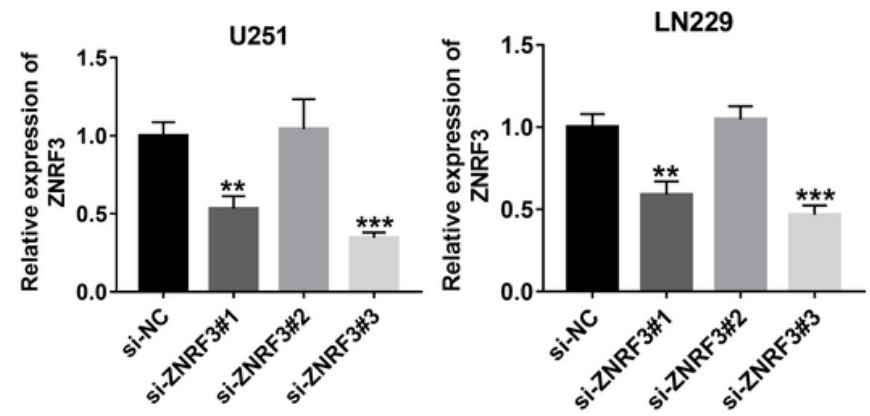

B

C

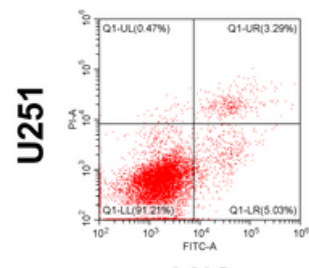

si-NC

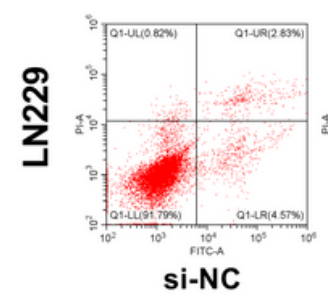

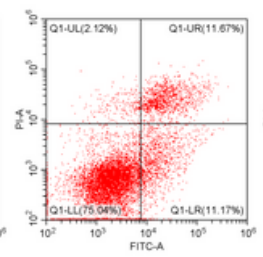

si-RMRP si-RMRP+si-ZNRF3

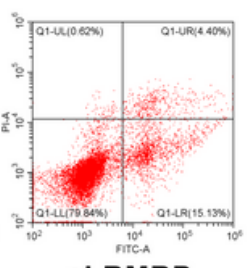

si-RMRP

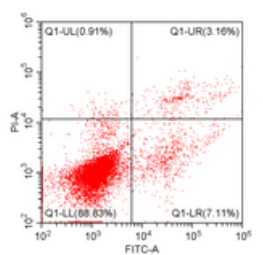

si-RMRP+si-ZNRF3
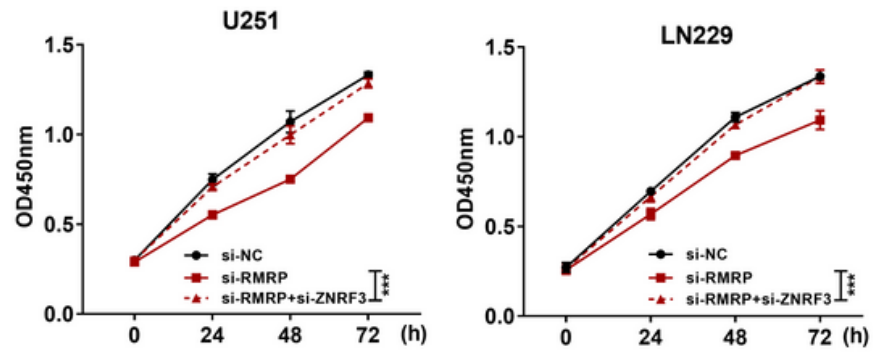

D

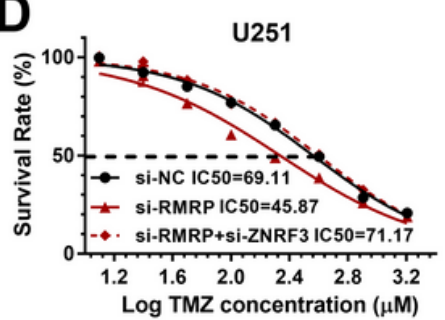

E
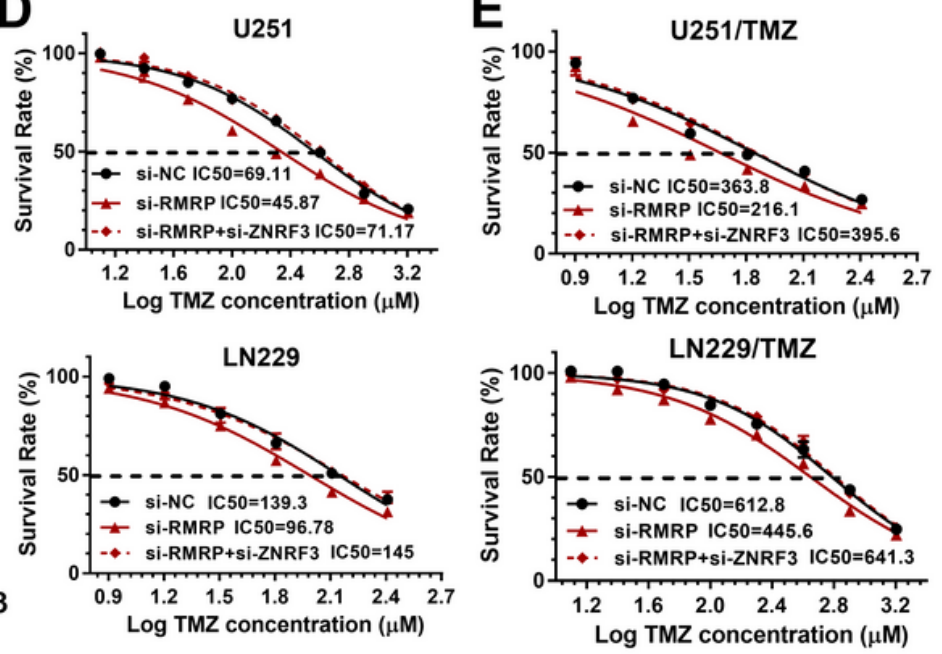

\section{Figure 5}

ZNRF3 down-regulation weakened the effects of RMRP knockdown on glioma cell proliferation, apoptosis and TMZ resistance. (A) Knockdown efficiency analysis of si-ZNRF3\#1, si-ZNRF3\#2, or siZNRF3\#3 in U251 and LN229 cells by RT-qPCR assay at $48 \mathrm{~h}$ post transfection. (B-D) U251 and LN229 cells were transfected with si-NC, si-RMRP, or si-RMRP+si-ZNRF3, followed by the measurement of cell proliferative ability (B), cell apoptotic rate (C) and TMZ IC50 values (D). (E) TMZ IC50 values were determined by CCK-8 assay in U251/TMZ and LN229/TMZ cells treated with different doses of TMZ. ${ }^{\star} * \mathrm{P}<0.01$. *** $\mathrm{P}<0.001$. 
A

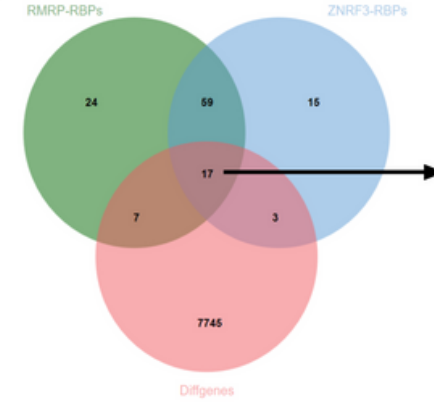

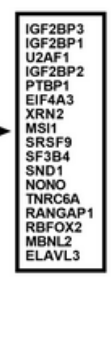

B

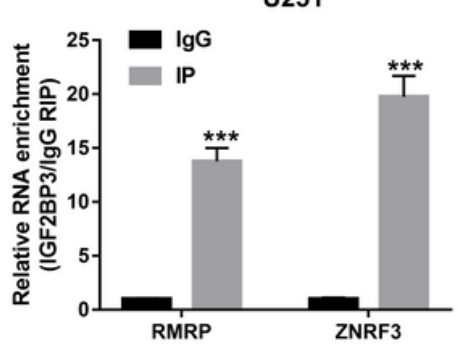

C

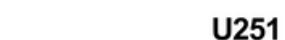

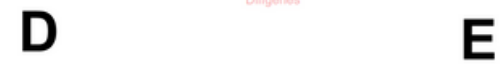

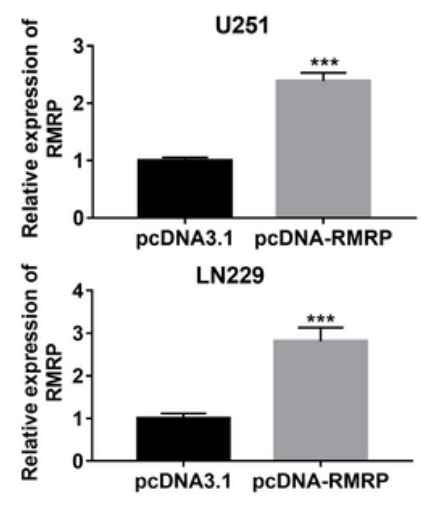

$\mathbf{E}$

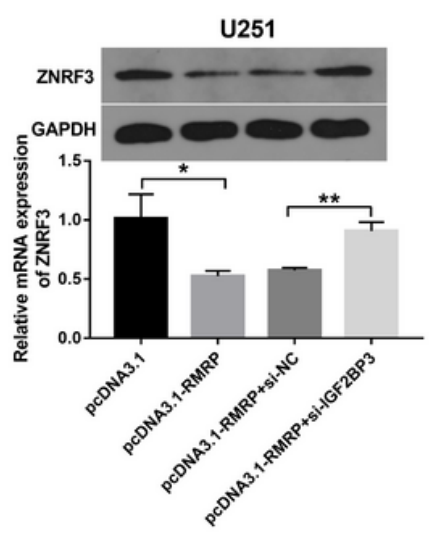

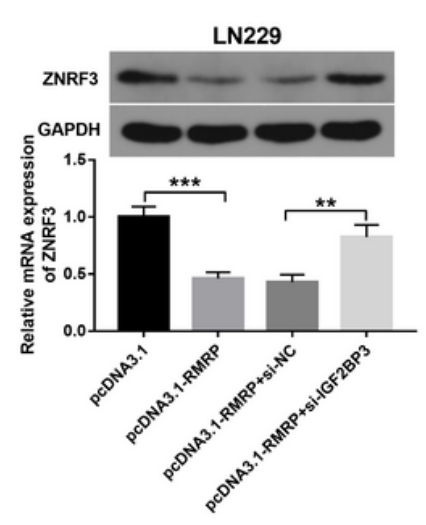

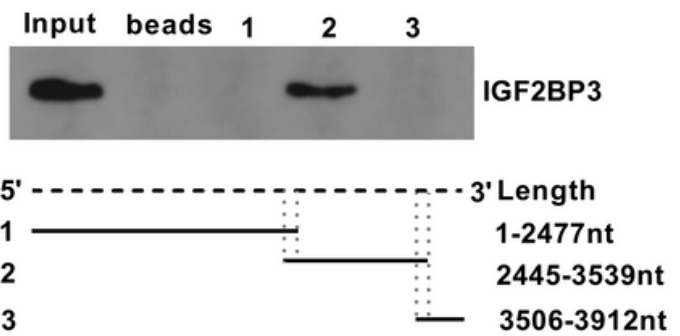

$\mathbf{F}$
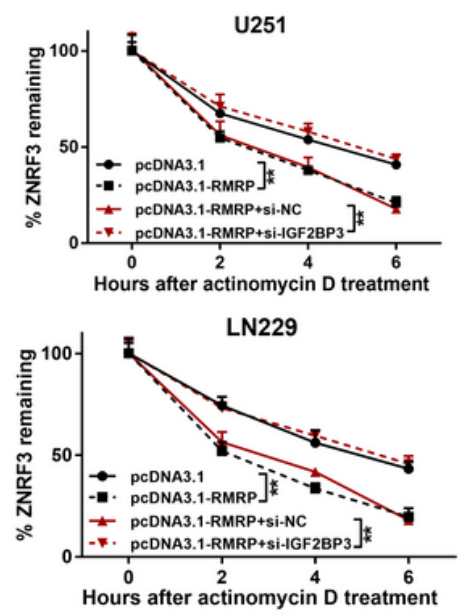

Figure 6

RMRP regulated ZNRF3 expression and ZNRF3 mRNA stability through RBP IGF2BP3. (A) Venn analysis for RMRP-RBPs, ZNRF3-RBPs and differentially expressed genes to screen out differentially expressed RBPs that had the possibility to bind with both RMRP and ZNRF3. RMRP-RBPs (green): RBPs with the possibility to bind with RMRP (predicted by POSTAR database). ZNRF3-RBPs (blue): RBPs with the possibility to bind with ZNRF3 mRNA (predicted by POSTAR database). Diffgenes (red): Differentially expressed genes in glioma (data were downloaded from TCGA/GTEx databases). (B) RIP assay was performed using IgG or IGF2BP3 antibody in U251 cells. Next, RMRP and ZNRF3 mRNA levels enriched by IgG or IGF2BP3 antibody were measured by RT-qPCR assay. (C) RNA pull down assay was performed using biotin-labeled ZNRF3 mRNA 3'UTR fragment 1, fragment 2 and fragment 3 in U251 cells, followed by the detection of IGF2BP3 protein level via western blot assay. (D) U251 and LN229 cells were transfected with pcDNA3.1 or pcDNA3.1-RMRP. Forty-eight hours later, RMRP level was measured by RTqPCR assay. (E and F) U251 and LN229 cells were transfected with pcDNA3.1, pcDNA3.1-RMRP, pcDNA3.1-RMRP+si-NC, or pcDNA3.1-RMRP+si-IGF2BP3. (E) ZNRF3 mRNA and protein levels were determined by RT-qPCR and western blot assays. (F) ZNRF3 mRNA stability was tested by Actinomycin D and RT-qPCR assays. ${ }^{*} P<0.01$. ${ }^{*} * \mathrm{P}<0.001$. 
A

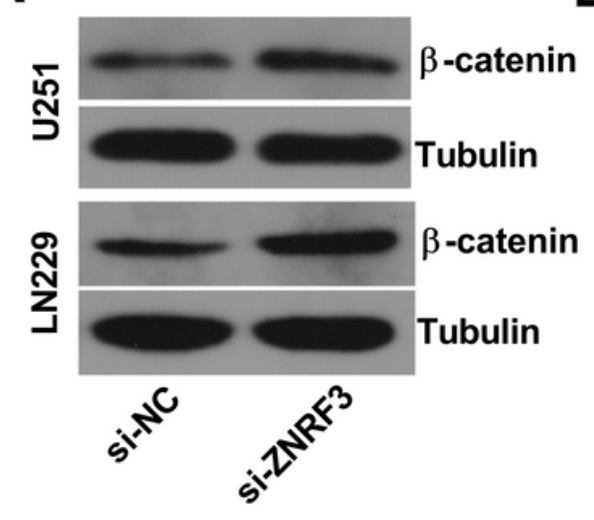

D

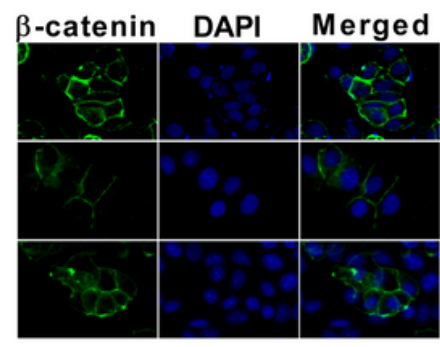

U251

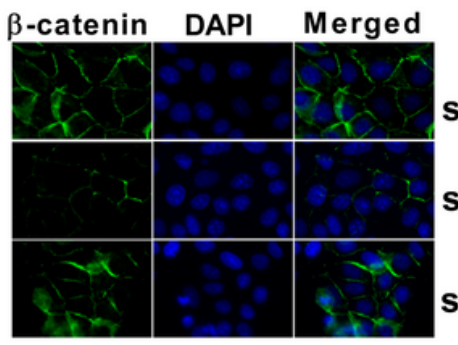

LN229

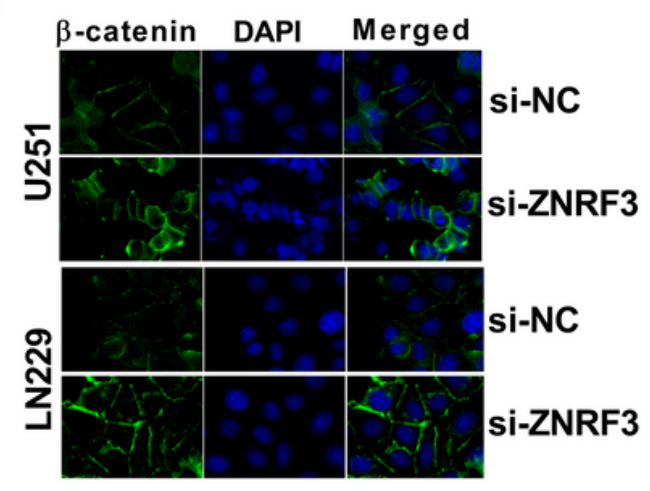

C
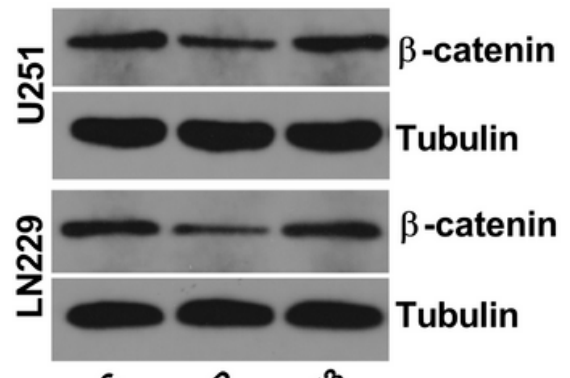

E

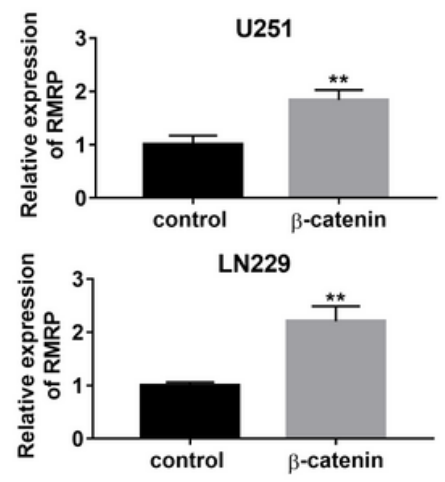

\section{Figure 7}

RMRP knockdown inhibited $\beta$-catenin expression by up-regulating ZNRF3 and $\beta$-catenin promoted RMRP expression in glioma cells. ( $A$ and $B$ ) Cells were transfected with si-NC or si-ZNRF3 for $48 \mathrm{~h}$, followed by the measurement of $\beta$-catenin protein level via western blot assay (A) and IF assay (B). (C and D) Cells were transfected with si-NC, si-RMRP or si-RMRP+si-ZNRF3. At $48 \mathrm{~h}$ post transfection, western blot assay (C) and IF assay (D) were performed to examine the protein expression pattern of $\beta$-catenin. (E) The effect of $\beta$-catenin overexpression or not on RMRP expression was examined by RT-qPCR assay in U251 and LN229 cells. ${ }^{\star *} \mathrm{P}<0.01$. 